\title{
Stability of downflowing gyrotactic microorganism suspensions in a two-dimensional vertical channel
}

\author{
YONGYUN HWANG ${ }^{1} \dagger$ AND T. J. PEDLEY
}

${ }^{1}$ Department of Civil and Environmental Engineering, Imperial College London, South Kensington, London SW7 2AZ, UK

${ }^{2}$ Department of Applied Mathematics and Theoretical Physics, University of Cambridge, Centre for Mathematical Sciences, Wilberforce Road, Cambridge CB3 0WA, UK

(Received 29 April 2014 and in revised form ??)

Hydrodynamic focusing of cells along the region of the most rapid flow is a robust feature in downflowing suspensions of swimming gyrotactic microorganisms. Experiments performed in a downward pipe flow have reported that the focussed beam-like structure of the cells is often unstable and results in the formation of regular-spaced axisymmetric blips, but the mechanism by which they are formed has not been well understood. To elucidate this mechanism, in this study, we perform a linear stability analysis of a downflowing suspension of randomly swimming gyrotactic cells in a two-dimensional vertical channel. On increasing the flow rate, the basic state exhibits a focussed beam-like structure. It is found that this focussed beam is unstable with the varicose mode, the spatial structure, wavelength, phase speed, and behaviour with the flow rate of which are remarkably similar to those of the blip instability in the pipe flow experiment. To understand the physical mechanism of the varicose mode, we perform an analysis which calculates the term-by-term contribution to the instability. It is shown that the leading physical mechanism in generating the varicose instability originates from the horizontal gradient in the cell-swimming-vector field formed by the non-uniform shear in the base flow. This mechanism is found to be supplemented by cooperation with the gyrotactic instability mechanism observed in uniform suspensions.

\section{Introduction}

Gyrotaxis is the term that describes the biased swimming of microorganisms such as Chlamydomonas, Dunaliella, and Volvox in the presence of shear in the surrounding fluid (Kessler 1984). These microorganisms are structurally bottom-heavy: i.e. their centre of mass is located behind the centre of buoyancy. Therefore, when a microorganism of this type is not aligned with the vertical, the bottom-heaviness generates a gravitational torque which reorients the cell to the vertical. This mechanism leads the microorganism to be naturally gravitactic: it swims against gravity in the absence of shear. However, when a shear is imposed in the surrounding fluid, the microorganism also experiences a viscous torque due to the shear. Therefore, the orientation of the cell is determined by the balance between the gravitational and the viscous torques, and the term 'gyrotaxis' refers to this process (Kessler 1984, 1985a,b, 1986).

In addition to determining the swimming orientation of a single cell, gyrotaxis plays

$\dagger$ E-mail address for correspondence: y.hwang@imperial.ac.uk 
a crucial role in generating instabilities in suspensions of bottom-heavy microorganisms (Pedley \& Kessler 1992; Hill \& Pedley 2005; Pedley 2010b). The stationary bottomstanding streaky plumes observed in a deep and uniform suspension $(d>1 \mathrm{~cm}$ where $d$ is the depth of the suspension) are an example of the gyrotactic instability (see e.g. Kessler 1986; Pedley \& Kessler 1992). We suppose a small 'blob' of higher concentration of cells in an otherwise uniform suspension. As the blob is heavier than the surroundings, it will sink while generating a downward shear flow in its wake. Due to the gyrotactic nature of the bottom-heavy cell, the downward shear flow leads the other cells to swim toward the blob and its wake. The blob therefore becomes denser and creates even stronger downflow, causing an instability in the suspension (Pedley et al. 1988; Pedley \& Kessler 1990; Pedley 2010a,b). Another example of the crucial role played by gyrotaxis in generating instability is bioconvection which appears in a shallow layer $(d<1 \mathrm{~cm})$ of the suspension (Childress et al. 1975; Kessler 1985a; Hill et al. 1989; Bees \& Hill 1998; Hwang \& Pedley 2014). Here, the gravitactic up-swimming cells quickly accumulate at the top end of the suspension and form a layer of cells heavier than the surroundings. This leads the suspension to experience a gravitational instability and generates a flow pattern similar to that of Rayleigh-Bénard convection (Pedley \& Kessler 1992; Bees \& Hill 1997). However, contrary to the classical Rayleigh-Bénard convection, the pattern in bioconvection is not a simple consequence of the gravitational instability. As recently shown by the authors (Hwang \& Pedley 2014), the gyrotactic instability mechanism also plays an important cooperative role in generating the instability (particularly at high wavenumbers). Furthermore, under an unstably stratified background cell concentration, the gyrotaxis yields non-negligible amounts of cross-diffusion flux to locally denser regions of the cells, which also significantly contributes to the bioconvective instability (Hwang \& Pedley 2014).

The instabilities observed in the suspensions of gyrotactic cells are often a consequence of cooperation and/or competition of multiple physical processes, and this feature often hampers precise understanding of the origin of the instabilities. An example of this is the blip instability observed in the early experiment of Kessler (1984, 1985a,b, 1986) which demonstrated gyrotaxis of the bottom-heavy cells. The experiment is composed of a vertical pipe in which a downward through flow of the cell suspension is applied. Kessler then demonstrated the gyrotaxis by showing the formation of a vertical beam-like structure along the pipe axis as a consequence of the accumulation of the bottom-heavy cells in the region of most rapid downward flow. In this experiment, interestingly, he reported that the vertical beam exhibits an instability in the form of regularly-spaced axisymmetric blips, each of which eventually develops into a vortex ring further downstream (Kessler $1985 a, 1986)$. The typical spacing between the blips was $O(1 \mathrm{~cm})$ and each of them was found to fall faster than the bulk fluid. He also reported that the blips predominantly appear at relatively low flow rates and that they gradually disappear on increasing the flow rate. This experiment was recently repeated by Denissenko \& Lukaschuk (2007) and they reported that the blips are also suppressed at very low flow rates.

Despite the relatively well-documented features of the blip instability, its origin has remained poorly understood. The early review by Pedley \& Kessler (1992) speculated that at least two mechanisms would be involved, one of which is from the gravity-driven instability observed in a dense falling plume through a surrounding fluid (Lister 1987; Smith 1989), and the other is associated with the inflection points in the velocity profile of the downflowing suspension caused by the strong gravitational force along the beam-like structure (see also a further discussion in §4.2). Recently, Denissenko \& Lukaschuk (2007) performed a simple analysis on the blip instability by deriving a system of equations for the width and the density of the axial beam. However, their analysis is too simple and 


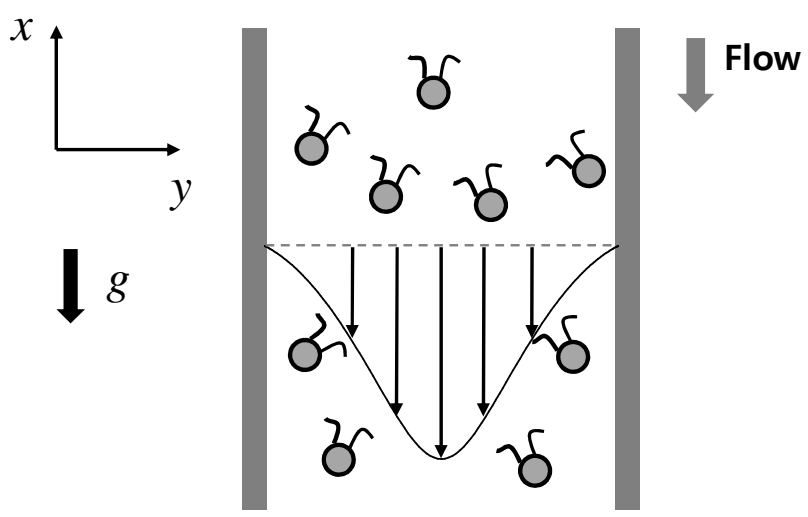

FIgURE 1. Schematic diagram of flow configuration in the present study.

is based on a number of assumptions that are not fully justified: for example, timedependence of the momentum equation of the fluid is ignored, and translational and rotational diffusion of the cells are completely neglected. Furthermore, the analysis does not provide any information on the spatial spacing of the blips as it assumes that the solution varies slowly along the vertical direction.

The goal of the present study is to gain fundamental understanding of the origin of the blip instability within a theoretical framework. We start with a more complete form of mathematical model developed by Pedley (2010a) where the distribution of the randomly swimming cells in time, space, and the cell swimming orientation-space is described with a probability density function satisfying a Smoluchowski equation. We then follow the approximations given in Hwang \& Pedley (2014) so that the computational cost of the present study is manageable. For simplicity, the flow configuration is set as a twodimensional vertical channel instead of the circular pipe (see figure 1). Although this configuration is not exactly the same as that of the experiment by Kessler $(1985 \mathrm{a})$ and Kessler (1986), we will see that many features of the instability found in the present study are remarkably similar to the blips observed in the pipe experiment. Finally, we should emphasise that the present study should be distinguished from previous work by Ghorai \& Hill $(1999,2000)$ who performed a linear stability analysis of naturally arising 'standing' gyrotactic plumes. In the present study, the basic state, obtained in the form of the beam-like structure as in the experiment, is essentially caused by the imposed flow. Therefore, the basic state given here is continuously connected to the uniform suspension of the cells in the absence of the imposed flow (see §3.1). In contrast, the basic state in Ghorai \& Hill $(1999,2000)$ was given in the form of a vertically uniform stationary plume, generated as a consequence of the gyrotactic instability in a stationary suspension, the top and bottom ends of which are closed by the no-fluid- and no-cell-flux conditions. In this respect, their analysis is precisely a secondary stability analysis of a uniform suspension in the given flow configuration (i.e. stability of the state bifurcated via a primary instability), which clearly differs from the present analysis. It should finally be mentioned that this interpretation is consistent with Bees \& Hill (1999) where the emergence and stability of stationary gyrotactic plumes in an unbounded and uniform suspension are analysed.

This paper is organised as follows: In $\S 2$, the equations of motion are introduced and formulated for a linear stability analysis. Parameters and numerical methods used in the present study are then given. In $\S 3$, the basic states are presented, and their linear stability is examined. In $\S 4$, we compare the present results with the previous laboratory 
experiments, and provide a detailed discussion on the origin of the blip instability using a budget analysis.

\section{Problem formulation}

\subsection{Equation of motion}

We consider a downward fluid flow in a vertical two-dimensional channel in which swimming gyrotactic microorganisms are suspended (see figure 1). We denote $x^{*}$ and $y^{*}$ as the vertical and horizontal directions, respectively, and $t^{*}$ is the time (the superscript * indicates dimensional variables). The suspension is assumed to be dilute (the average volume fraction of cells is typically less than 1\%), and is bounded by the two walls located at $y^{*}= \pm h$, respectively. The fluid in the suspension has density $\rho$ and kinematic viscosity $\nu$, and gravity heads downward in the $-x^{*}$ direction. The suspended microorganisms are assumed to be spherical 'puller'-type swimmers, which generate thrust by pulling fluid from the front to the back (e.g. C. nivalis, Dunaliella, and Volvox) as in the experiment of Kessler (1986). Their swimming speed is $V_{c}^{*}$ and they are assumed to perform random walks as a result of repeated reorientations. Following Hwang \& Pedley (2014), where a more detailed discussion of the present model is given, the equations of motion are then written as

$$
\begin{gathered}
\nabla^{*} \cdot \mathbf{u}^{*}=0, \\
\frac{\partial \mathbf{u}^{*}}{\partial t^{*}}+\left(\mathbf{u}^{*} \cdot \nabla^{*}\right) \mathbf{u}^{*}=-\frac{1}{\rho} \nabla^{*} p^{*}+\nu \nabla^{2} \mathbf{u}^{*}-n^{*} v g^{\prime} \mathbf{i}, \\
\frac{\partial n^{*}}{\partial t^{*}}+\nabla \cdot\left[n^{*}\left(\mathbf{u}^{*}+V_{c}^{*}\langle\mathbf{e}\rangle\right)\right]=\nabla^{*} \cdot\left(\mathbf{D}_{T}^{*} \cdot \nabla^{*} n^{*}\right),
\end{gathered}
$$

with boundary conditions

$$
\begin{gathered}
\left.\mathbf{u}^{*}\right|_{y^{*}= \pm h}=(0,0), \\
{\left.\left[n^{*}\left(\mathbf{u}^{*}+V_{c}^{*}\langle\mathbf{e}\rangle\right)-\mathbf{D}_{T}^{*} \cdot \nabla^{*} n^{*}\right]\right|_{y^{*}= \pm h} \cdot \mathbf{j}=0 .}
\end{gathered}
$$

Here, $\mathbf{u}^{*}$ is the velocity, $p^{*}$ the pressure, $n^{*}$ the cell number density, $g^{\prime}=g \Delta \rho / \rho$ the reduced gravity ( $g$ and $\Delta \rho$ are the gravitational acceleration and the density difference between cell and fluid, respectively), $v$ the volume of a single cell, e the unit vector indicating the swimming direction, $\mathbf{D}_{T}^{*}$ the diffusivity tensor for the cell number density, and $\mathbf{i}$ and $\mathbf{j}$ are respectively the unit vectors in the vertical and wall-normal directions. The no-slip condition $(2.1 d)$ is imposed at the wall for the fluid velocity, and the no-flux condition $(2.1 e)$ is applied for the cell-number density so that total number of the cells is preserved in time.

In $(2.1 c),\langle\cdot\rangle$ indicates the local ensemble average at given $\mathbf{x}^{*}$ and time $t^{*}$ to take the random walk of the cell into account: for example, the mean swimming vector $\langle\mathbf{e}\rangle$ implies

$$
\langle\mathbf{e}\rangle\left(\mathbf{x}^{*}, t^{*}\right) \equiv \int_{\|\mathbf{e}\|=1} \mathbf{e} f\left(\mathbf{x}^{*}, \mathbf{e}, t^{*}\right) d^{2} \mathbf{e},
$$

where $f\left(\mathbf{x}^{*}, \mathbf{e}, t^{*}\right)$ is the probability density function (p.d.f.) for the cell orientation, satisfying

$$
\int_{\|\mathbf{e}\|=1} f\left(\mathbf{x}^{*}, \mathbf{e}, t^{*}\right) d^{2} \mathbf{e}=1 .
$$

Under the assumption that the random walk is Brownian, the p.d.f obeys the following 
equation (Hwang \& Pedley 2014):

$$
\frac{\partial f}{\partial t^{*}}+\left(\mathbf{u}^{*} \cdot \nabla\right) f+\nabla_{e} \cdot\left[\frac{1}{2 B}[\mathbf{i}-(\mathbf{i} \cdot \mathbf{e}) \mathbf{e}] f+\frac{1}{2} \boldsymbol{\Omega}^{*} \wedge \mathbf{e} f\right]=D_{R}^{*} \nabla_{e}^{2} f
$$

where $B=\nu \alpha_{\perp} / 2 g l$ is the gyrotactic time scale ( $l$ is the centre of gravity offset of the cell and $\alpha_{\perp}(=0.6)$ is a geometrical constant for the spherical cell), $\boldsymbol{\Omega}^{*}$ the vorticity, and $D_{R}^{*}$ the rotational diffusivity. We note that equations $(2.1 c)$ and $(2.2 c)$ are derived by approximating the Smoluchoski equation equation which gives the probability density function for distribution of the cell number density and the swimming orientation (Hwang $\&$ Pedley 2014). The approximation is valid if the length scale and the shear rate (or vorticity) of the system are sufficiently large: i.e. $h \gtrsim O(0.01 \mathrm{~cm})$ and $O\left(\mathbf{u}^{*} / h\right) \gtrsim O\left(B^{-1}\right)$. However, this does not limit the present study as the experiments of Kessler $(1985 a, 1986)$ were performed under such conditions. Finally, in the present study, the cell swimming in $(2.2 c)$ is fully three dimensional (i.e. $\mathbf{e}=\left(e_{1}, e_{2}, e_{3}\right)$ ) although the flow field is considered to be two-dimensional. In this respect, the present study is precisely an analysis of twodimensional dynamics in a three-dimensional vertical channel.

For the diffusivity tensor in $(2.1 c)$, we use the simplified expression given in Pedley \& Kessler (1990):

$$
\mathbf{D}_{T}^{*}=V_{c}^{* 2} \tau(\langle\mathbf{e e}\rangle-\langle\mathbf{e}\rangle\langle\mathbf{e}\rangle)
$$

where $\tau$ is the correlation time scale for the cell orientation. The assumption of a constant $\tau$ may not be a reasonable description particularly if the local vorticity (or the flow rate) is large (see also e.g. Bearon et al. 2012; Croze et al. 2013). It can be improved by the generalised Taylor dispersion theory as recently addressed (Hill \& Bees 2002; Malena \& Frankel 2003; Bearon et al. 2011). However, it should also be pointed out that it is not evident yet whether the generalised Taylor dispersion theory itself would provide a good quantitative prediction for $\mathbf{D}_{T}^{*}$ in 'real' cell suspensions. An important assumption in the generalised Taylor dispersion theory, as also in the present study, is that the rotational diffusivity does not change with the local vorticity (see e.g. Hill \& Bees 2002; Malena \& Frankel 2003). However, in reality, this does not seem to be true: the rotational diffusivity does depend on the vorticity (S. Furlan, T. J. Pedley \& R. E. Goldstein 2014, unpublished observation). The underlying difficulty caused by the lack of an experimentally verified expression for $\mathbf{D}_{T}^{*}$ leads us still to rely on the simple expression (2.3) and to perform a parametric study with respect to the correlation time $\tau$ and the rotational diffusivity $D_{R}^{*}$ instead (see $\S 3.3)$.

\subsection{Non-dimensionalization}

The governing equation (2.1) is made dimensionless by introducing

$$
x=\frac{x^{*}}{h}, y=\frac{y^{*}}{h}, t=\frac{t^{*} V_{c}}{h}, \mathbf{u}=\frac{\mathbf{u}^{*}}{V_{c}}, p=\frac{p^{*}}{\rho V_{c}^{* 2}}, n=\frac{n^{*}}{N},
$$

where $N=1 / V \int_{\Omega} n^{*} d V$ and $\Omega$ is the domain of interest, with volume $V$. We note that the velocity scale is chosen to be $V_{c}^{*}$ instead of the one associated with the translational diffusion flux $\left(V_{c}^{* 2} \tau / h\right)$ in our previous study (Hwang \& Pedley 2014). The dimensionless form of (2.1) is then given as

$$
\begin{gathered}
\nabla \cdot \mathbf{u}=0 \\
\frac{\partial \mathbf{u}}{\partial t}+(\mathbf{u} \cdot \nabla) \mathbf{u}=-\nabla p+\frac{1}{\operatorname{Re}} \nabla^{2} \mathbf{u}-\operatorname{Ri} n \mathbf{i}, \\
\frac{\partial n}{\partial t}+\nabla \cdot[n(\mathbf{u}+\langle\mathbf{e}\rangle)]=\frac{1}{\operatorname{ScRe}} \nabla \cdot\left(\mathbf{D}_{T} \cdot \nabla n\right),
\end{gathered}
$$




$$
D_{R}^{-1} \frac{\partial f}{\partial t}+D_{R}^{-1}(\mathbf{u} \cdot \nabla) f+\nabla_{e} \cdot\left[\lambda[\mathbf{i}-(\mathbf{i} \cdot \mathbf{e}) \mathbf{e}] f+\frac{1}{2 D_{R}} \boldsymbol{\Omega} \wedge \mathbf{e} f\right]=\nabla_{e}^{2} f,
$$

with boundary conditions

$$
\begin{gathered}
\left.\mathbf{u}\right|_{y= \pm 1}=(0,0), \\
{\left.\left[n\left(\mathbf{u}+V_{c}\langle\mathbf{e}\rangle\right)-\frac{1}{\operatorname{ScRe}} \mathbf{D}_{T} \cdot \nabla n\right]\right|_{y= \pm 1} \cdot \mathbf{j}=0}
\end{gathered}
$$

where

$$
\operatorname{Re}=\frac{V_{c}^{*} h}{\nu}, \mathrm{Ri}=\frac{N v g^{\prime} h}{V_{c}^{* 2}}, \mathrm{Sc}=\frac{\nu}{V_{c}^{* 2} \tau}, \mathbf{D}_{T}=\frac{\mathbf{D}_{T}^{*}}{V_{c}^{* 2} \tau}, \lambda=\frac{1}{2 B D_{R}^{*}}, D_{R}=\frac{D_{R}^{*} h}{V_{c}^{*}} .
$$

Here, Re is the Reynolds number based on the cell swimming velocity, Ri the Richardson number indicating the ratio of the potential energy of the suspension to the kinetic energy caused by the swimming motion, Sc the Schmidt number representing the ratio of the kinematic viscosity to the translational diffusivity of the cell number density, $\lambda$ the inverse of dimensionless gyrotactic time scale, and $D_{R}$ the dimensionless rotational diffusivity. We note that the Péclet number based on the cell-swimming velocity $V_{c}^{*}$ is then defined as $\mathrm{Pe}=$ ScRe.

\subsection{Basic state}

We first calculate the basic state of (2.5) (i.e. the steady solution), to which a small perturbation will be added for a linear stability analysis. Since the flow configuration is homogeneous along the vertical direction, the velocity and the cell number density for the basic state satisfy

$$
\frac{\partial}{\partial x}=0, \mathbf{u}=\mathbf{u}_{0}\left(=\left(U_{0}(y), 0\right)\right), n=n_{0}(y)
$$

yielding

$$
\begin{gathered}
-\frac{\partial P_{0}}{\partial x}+\frac{1}{\operatorname{Re}} \frac{d^{2} U_{0}}{d y^{2}}-\operatorname{Ri} n_{0}=0, \\
-\frac{\partial P_{0}}{\partial y}=0, \\
\frac{d}{d y}\left[-n_{0}\left\langle e_{2}\right\rangle_{0}+\frac{1}{\operatorname{ScRe}} D_{T 0}^{22} \frac{d n_{0}}{d y}\right]=0, \\
\nabla_{e} \cdot\left[\lambda[\mathbf{i}-(\mathbf{i} \cdot \mathbf{e}) \mathbf{e}] f_{0}+\frac{1}{2 D_{R}} \boldsymbol{\Omega}_{0} \wedge \mathbf{e} f_{0}\right]=\nabla_{e}^{2} f_{0},
\end{gathered}
$$

with boundary conditions

$$
\begin{gathered}
\left.U_{0}\right|_{y= \pm 1}=0 \\
-\left.n_{0}\left\langle e_{2}\right\rangle_{0}\right|_{y= \pm 1}+\left.\frac{1}{\operatorname{ScRe}} D_{T 0}^{22} \frac{d n_{0}}{d y}\right|_{y= \pm 1}=0
\end{gathered}
$$

Here, $P_{0}$ is the basic-state pressure and $\boldsymbol{\Omega}_{0}$ is the basic state vorticity, and the subscript 0 in $\left\langle e_{2}\right\rangle_{0}$ and $D_{T 0}^{22}$ implies the statistical properties obtained with $f_{0}$. From $(2.7 a)$ and $(2.7 b), \partial P_{0} / \partial x$ is constant in $t$ and $y$. Therefore, assuming even symmetry of the basic state (i.e. $U_{0}(y)=U_{0}(-y)$ and $n_{0}(y)=n_{0}(-y)$; see also figure 4), integration of $(2.7 a)$ over $y \in[-1,0]$ yields

$$
\frac{d P_{0}}{d x}=-\left.\frac{1}{\operatorname{Re}} \frac{d U_{0}}{d y}\right|_{y=-1}+\mathrm{Ri}
$$



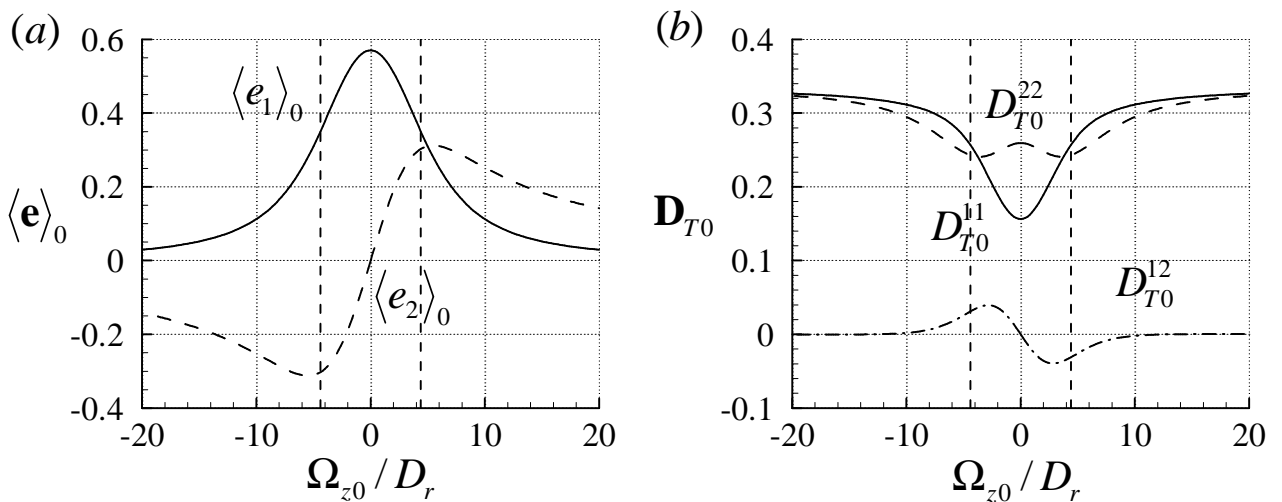

Figure 2. (a) Mean cell swimming vector and $(b)$ diffusivity tensor $(\lambda=2.2)$. Here, the dashed vertical lines indicate $\Omega_{z 0}= \pm 4.4 D_{r}$ at which the deterministic swimmer begins to tumble.

This equation indicates that the vertical pressure gradient $d P_{0} / d x$ is composed of two parts: one is the driving pressure gradient to generate a downward flow (the first term on the right-hand side) and the other is the hydrostatic pressure required to balance the gravitational force caused by the negatively buoyant cells (the second term on the righthand side). It is important to note that the hydrostatic part of the pressure gradient is independent of the flow rate as $\mathrm{Ri}$ is given by the properties of the cells and the flow geometry. Therefore, for a given flow configuration, the hydrostatic part $d P_{0}^{h} / d x=\mathrm{Ri}$ can be separated from $d P_{0} / d x$, leading to

$$
-\frac{d P_{0}^{d}}{d x}+\frac{1}{\operatorname{Re}} \frac{d^{2} U_{0}}{d y^{2}}-\operatorname{Ri}\left(n_{0}-1\right)=0,
$$

where $d P_{0}^{d} / d x\left(=1 / \operatorname{Re} d U_{0} /\left.d y\right|_{y=-1}\right)$ is the pressure gradient for driving the flow. We also note that (2.9) provides a physically consistent solution of (2.7) for the stationary case: i.e. $U_{0}(y)=0$ and $n_{0}(y)=1$ (uniform suspension) if $d P_{0}^{d} / d x=0$.

In obtaining the solution of (2.7), it is also important to note that $(2.7 d)$ does not contain any partial derivative in $\mathbf{x}$. This implies that $f_{0}(\mathbf{x}, \mathbf{e})$ is not explicitly dependent upon the spatial location $\mathbf{x}$, and only depends on the local vorticity $\Omega_{0}\left(=\Omega_{z 0} \mathbf{k}\right.$ where $\left.\Omega_{z 0}=-d U_{0} / d y\right)$. Therefore, once $\langle\mathbf{e}\rangle_{0}$ and $\mathbf{D}_{T 0}$ are obtained in terms of $\boldsymbol{\Omega}_{0}$ by solving $(2.7 d)$, they can be used as functions of $\boldsymbol{\Omega}_{0}$ for $(2.7 c)$. Numerical solution of $(2.7 d)$ for a given local vorticity (or shear rate) has been obtained in detail in our previous study (Hwang \& Pedley 2014). Therefore, in the present study, we briefly report how $\langle\mathbf{e}\rangle_{0}$ and $\mathbf{D}_{T 0}$ behave with the local vorticity $\Omega_{z 0}$ (see figure 2 ). In the absence of the vorticity $\left(\Omega_{z 0}=0\right)$, the mean swimming orientation $\langle\mathbf{e}\rangle_{0}$ heads upward (figure $2 a$ ) and the diffusivity tensor $\mathbf{D}_{T 0}$ becomes highly anisotropic (figure $2 b$ ) due to the gravitaxis. As $\Omega_{z 0}$ is increased, but while it is small (i.e. $0<\left|\Omega_{z 0} / D_{R}\right|<4 \sim 5$ ), the mean swimming vector is gradually tilted along the rotational direction of the given vorticity, and the cross component of $\mathbf{D}_{T 0}$ (i.e. $D_{T 0}^{12}$ ) is also generated. However, if the strength of the vorticity is sufficiently large $\left(\left|\Omega_{z 0} / D_{R}\right|>4 \sim 5\right)$, this tendency is changed. In this case, the size of the mean swimming vector $\left(\left|\langle\mathbf{e}\rangle_{0}\right|\right)$ decays and the diffusivity tensor tends towards isotropicity as the vorticity strength is increased. It is worth noting that this change is seen to appear from $\left|\Omega_{z 0} / D_{R}\right| \simeq 4$.4, the value at which a deterministic swimmer would begin to tumble.

In general, the nonlinear dependence of $\langle\mathbf{e}\rangle_{0}$ and $\mathbf{D}_{T 0}$ on $\Omega_{z 0}$ and the two-way coupling between $(2.7 c)$ and (2.9) yield a difficulty in seeking a general analytic solution of $(2.7)$, 
except for $\mathrm{Ri}=0$ which admits the following solution:

$$
\begin{gathered}
U_{0}(y)=\frac{\operatorname{Re}}{2} \frac{d P_{0}^{d}}{d x}\left(y^{2}-1\right), \\
n_{0}(y)=N_{0} e^{\operatorname{ScRe} \int \frac{\left\langle e_{2}\right\rangle_{0}}{D_{T 0}^{22}} d y,}
\end{gathered}
$$

where $N_{0}$ is a normalization constant giving $1 / V \int_{\Omega} n_{0}(y) d V=1$. We note that this is simply a plane Poiseulle flow, and it can occur if the cells are neutrally buoyant. For $\mathrm{Ri} \neq 0$, the solution of $(2.7)$ is obtained numerically as detailed in $\S 2.6$. In the present study, we perform the entire analysis by prescribing the flow rate,

$$
Q=-\int_{-1}^{1} U_{0}(y) d y
$$

with a given value rather than giving a fixed pressure gradient $d P_{0}^{d} / d x$ (see $\S 3.1$ for a detailed discussion). Using the flow rate $Q$, the Reynolds number based on the bulk flow velocity $U_{b}(=Q / 2)$ is obtained as

$$
\operatorname{Re}_{b}=\frac{Q \operatorname{Re}}{2}
$$

where the factor 2 in the denominator originates from the channel width $(2 h)$.

\subsection{Linear stability analysis}

Now, we consider a small perturbation about the basic state:

$$
\begin{aligned}
& \mathbf{u}=\mathbf{u}_{0}(\mathbf{x})+\epsilon \mathbf{u}^{\prime}(\mathbf{x}, t)+O\left(\epsilon^{2}\right), p=P_{0}(\mathbf{x})+\epsilon p^{\prime}(\mathbf{x}, t)+O\left(\epsilon^{2}\right), \\
& n=n_{0}(\mathbf{x})+\epsilon n^{\prime}(\mathbf{x}, t)+O\left(\epsilon^{2}\right), f=f_{0}(\mathbf{e})+\epsilon f^{\prime}(\mathbf{x}, \mathbf{e}, t)+O\left(\epsilon^{2}\right),
\end{aligned}
$$

where $\mathbf{u}^{\prime}=\left(u^{\prime}, v^{\prime}\right)$ and $\epsilon \ll 1$. Here, we note that the perturbation of the vertical velocity and the cell number density over the given control volume should satisfy

$$
\int_{\Omega} u^{\prime} d V=0 \text { and } \int_{\Omega} n^{\prime} d V=0
$$

as the flow rate $Q$ is fixed and the total number of the cells over the entire domain is preserved in time. The linearised equations for the small perturbation are then given as

$$
\begin{gathered}
\frac{\partial u^{\prime}}{\partial x}+\frac{\partial v^{\prime}}{\partial y}=0, \\
\frac{\partial u^{\prime}}{\partial t}+U_{0} \frac{\partial u^{\prime}}{\partial x}+v^{\prime} \frac{\partial U}{\partial y}=-\frac{\partial p^{\prime}}{\partial x}+\frac{1}{\operatorname{Re}} \nabla^{2} u^{\prime}-\operatorname{Ri} n^{\prime}, \\
\frac{\partial v^{\prime}}{\partial t}+U_{0} \frac{\partial v^{\prime}}{\partial x}=-\frac{\partial p^{\prime}}{\partial y}+\frac{1}{\operatorname{Re}} \nabla^{2} v^{\prime}, \\
\frac{\partial n^{\prime}}{\partial t}+\left(U_{0}+\left\langle e_{1}\right\rangle_{0}\right) \frac{\partial n^{\prime}}{\partial x}+\left\langle e_{2}\right\rangle_{0} \frac{\partial n^{\prime}}{\partial y}+n^{\prime} \frac{d\left\langle e_{2}\right\rangle_{0}}{d y}+v^{\prime} \frac{d n_{0}}{d y} \\
+\left\langle e_{2}\right\rangle^{\prime} \frac{d n_{0}}{d y}+n_{0} \frac{\partial\left\langle e_{1}\right\rangle^{\prime}}{\partial x}+n_{0} \frac{\partial\left\langle e_{2}\right\rangle^{\prime}}{\partial y} \\
\frac{\operatorname{ScRe}}{D_{T 0}^{11}} \frac{\partial^{2} n^{\prime}}{\partial x^{2}}+2 D_{T 0}^{12} \frac{\partial^{2} n^{\prime}}{\partial x \partial y}+\frac{d D_{T 0}^{21}}{d y} \frac{\partial n^{\prime}}{\partial x}+D_{T 0}^{22} \frac{\partial^{2} n^{\prime}}{\partial y^{2}}+\frac{d D_{T 0}^{22}}{d y} \frac{\partial n^{\prime}}{\partial y} \\
\left.+\frac{\partial D_{T}^{12^{\prime}}}{\partial x} \frac{d n_{0}}{d y}+\frac{\partial D_{T}^{22^{\prime}}}{d y} \frac{d n_{0}}{\partial y}+D_{T}^{22^{\prime}} \frac{d^{2} n_{0}}{d y^{2}}\right],
\end{gathered}
$$



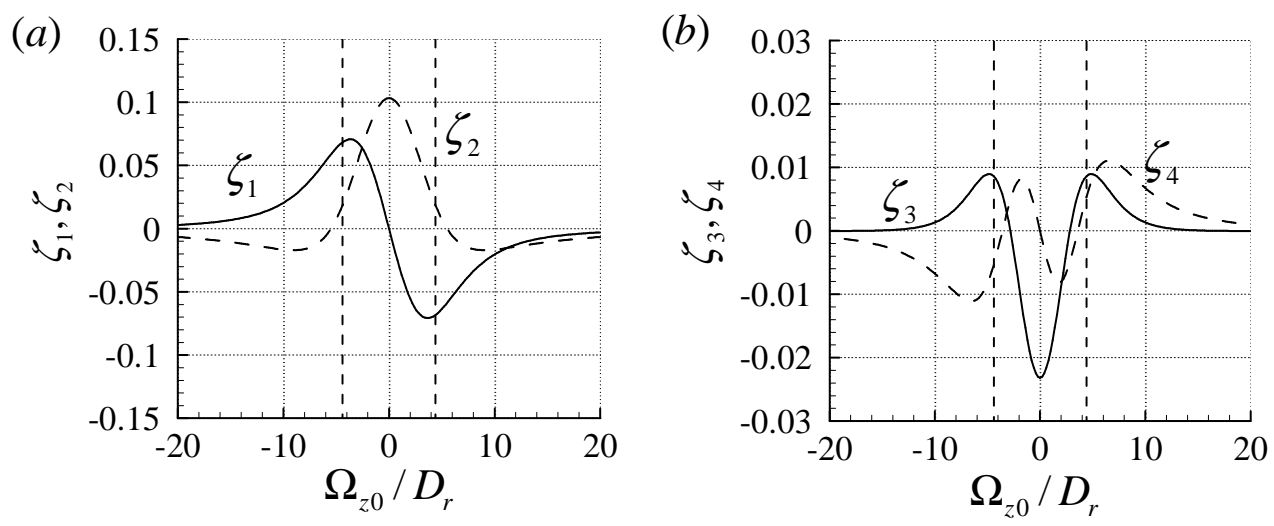

FiguRE 3. Dependence of $(a) \zeta_{1}, \zeta_{2}$ and $(b) \zeta_{3}, \zeta_{4}$ on $\Omega_{z}(\lambda=2.2)$. Here, the dashed vertical lines indicate $\Omega_{z 0}= \pm 4.4 D_{r}$ at which the deterministic swimmer begins to tumble.

$$
\begin{aligned}
& D_{R}^{-1} \frac{\partial f^{\prime}}{\partial t}+D_{R}^{-1} U_{0} \frac{\partial f^{\prime}}{\partial x}+\nabla_{e} \cdot\left[\lambda[\mathbf{j}-(\mathbf{j} \cdot \mathbf{e}) \mathbf{e}] f^{\prime}+\frac{1}{2 D_{R}} \boldsymbol{\Omega}_{0} \wedge \mathbf{e} f^{\prime}\right]-\nabla_{e}^{2} f^{\prime} \\
& =-D_{R}^{-1} \nabla_{e} \cdot\left[\frac{1}{2} \boldsymbol{\Omega}^{\prime} \wedge \mathbf{e} f_{0}\right]
\end{aligned}
$$

with boundary conditions

$$
\begin{gathered}
\left.u^{\prime}\right|_{y= \pm 1}=\left.v^{\prime}\right|_{y= \pm 1}=0 \\
{\left[\left\langle e_{2}\right\rangle_{0} n^{\prime}+\left\langle e_{2}\right\rangle^{\prime} n_{0}\right]-\left.\frac{1}{\operatorname{ScRe}}\left[D_{T 0}^{12} \frac{\partial n^{\prime}}{\partial x}+D_{T 0}^{22} \frac{\partial n^{\prime}}{\partial y}+D_{T}^{22^{\prime}} \frac{d n_{0}}{d y}\right]\right|_{y= \pm 1}=0,}
\end{gathered}
$$

where the superscript ' for $\left\langle e_{i}\right\rangle$ and $D_{T}^{i j}(i, j=1,2)$ indicates the statistical properties obtained from $f^{\prime}$. As discussed in Hwang \& Pedley (2014), performing a linear stability analysis directly with (2.15) would not be computationally feasible in practice due to the large dimension of (2.15) and the number of parameters to be studied. Therefore, here we approximate (2.15e) as in Hwang \& Pedley (2014) where $f^{\prime}$ is assumed to be quasi steady and quasi uniform: i.e.

$$
\nabla_{e} \cdot\left[\lambda[\mathbf{j}-(\mathbf{j} \cdot \mathbf{e}) \mathbf{e}] f^{\prime}+\frac{1}{2 D_{R}} \boldsymbol{\Omega}_{0} \wedge \mathbf{e} f^{\prime}\right]-\nabla_{e}^{2} f^{\prime}=-\frac{1}{2 D_{R}} \nabla_{e} \cdot\left[\boldsymbol{\Omega}^{\prime} \wedge \mathbf{e} f_{0}\right] .
$$

It is evident that the approximation is valid only if $f^{\prime}$ varies slowly in time and space: $f^{\prime}(t, \mathbf{x}, \mathbf{e})=f^{\prime}(T, \mathbf{X}, \mathbf{e})$ where $T=\delta t$ and $\mathbf{X}=\delta \mathbf{x}$ with $\delta \ll 1$. We note that the instabilities in the present study indeed appear in such situations (see §3.2), indicating that relaxing the approximation would not significantly modify the parameter range over which the instabilities appear. The approximated equation (2.16) allows us to write its solution as $f^{\prime}(t, \mathbf{x}, \mathbf{e})=D_{R}{ }^{-1} \omega_{z}^{\prime}(t, \mathbf{x}) f_{\omega_{z}}^{\prime}(\mathbf{e})$ where $\omega_{z}^{\prime}$ is the perturbed spanwise vorticity and $f_{\omega_{z}}^{\prime}(\mathbf{e})$ is the solution of $(2.16)$ if $\omega_{z}^{\prime}=1$ and $D_{R}=1$. Therefore, the statistical moments in $(2.15 d)$ such as $\left\langle e_{1}\right\rangle^{\prime},\left\langle e_{2}\right\rangle^{\prime}, D_{T}^{12^{\prime}}$, and $D_{T}^{22{ }^{\prime}}$ are given in the form of

$$
\left\langle e_{1}\right\rangle^{\prime}=\frac{1}{D_{R}} \omega_{z}^{\prime} \zeta_{1},\left\langle e_{1}\right\rangle^{\prime}=\frac{1}{D_{R}} \omega_{z}^{\prime} \zeta_{2}, D_{T}^{12^{\prime}}=\frac{1}{D_{R}} \omega_{z}^{\prime} \zeta_{3}, D_{T}^{22^{\prime}}=\frac{1}{D_{R}} \omega_{z}^{\prime} \zeta_{4},
$$

where $\zeta_{i}$ for $i=1,2,3,4$ are obtained from the first- and the second-order moments of $f_{\omega_{z}}^{\prime}(\mathbf{e})$ (for further details, see also Hwang \& Pedley 2014). The computed $\zeta_{i}$ using the numerically obtained $f_{\omega_{z}}^{\prime}(\mathbf{e})$ in Hwang \& Pedley (2014) are shown in figure 3.

In practice, the use of (2.16) instead of (2.15e) makes the present approach practically identical to that in Pedley \& Kessler (1990) where $\langle\mathbf{e}\rangle$ and $\mathbf{D}_{T}$ are generally obtained 
from the quasi-steady and quasi-uniform Fokker-Planck equation: i.e. (2.2c) without the convective derivative terms. Within this framework, $\langle\mathbf{e}\rangle$ and $\mathbf{D}_{T}$ on $\Omega_{z}$ become identical to $\langle\mathbf{e}\rangle_{0}$ and $\mathbf{D}_{T 0}$ on $\Omega_{z 0}$ given in figure 1. For two-dimensional fluid flows, as in the present study, this implies that $\langle\mathbf{e}\rangle^{\prime}$ and $\mathbf{D}_{T}^{\prime}$ are also obtained from a Taylor-series expansion of $\langle\mathbf{e}\rangle$ and $\mathbf{D}_{T}$ around $\langle\mathbf{e}\rangle_{0}$ and $\mathbf{D}_{T 0}$ : i.e.

$$
\begin{aligned}
\langle\mathbf{e}\rangle & =\langle\mathbf{e}\rangle_{0}+\left.\frac{\epsilon}{D_{R}} \frac{d\langle\mathbf{e}\rangle}{d \Omega}\right|_{\Omega=\Omega_{0}} \omega_{z}^{\prime}+O\left(\epsilon^{2}\right), \\
\mathbf{D}_{T} & =\mathbf{D}_{T 0}+\left.\frac{\epsilon}{D_{R}} \frac{d \mathbf{D}_{T}}{d \Omega}\right|_{\Omega=\Omega_{0}} \omega_{z}^{\prime}+O\left(\epsilon^{2}\right),
\end{aligned}
$$

where $\langle\mathbf{e}\rangle_{0}=\left.\langle\mathbf{e}\rangle\right|_{\Omega_{z}=\Omega_{z 0}}$ and $\mathbf{D}_{T 0}=\left.\mathbf{D}_{T 0}\right|_{\Omega_{z}=\Omega_{z 0}}$. Therefore, $\zeta_{i}$ are simply given as

$$
\begin{gathered}
\zeta_{1}=\left.\frac{d\left\langle e_{1}\right\rangle}{d \Omega_{z}}\right|_{\Omega_{z}=\Omega_{z 0}}, \zeta_{2}=\left.\frac{d\left\langle e_{2}\right\rangle}{d \Omega_{z}}\right|_{\Omega_{z}=\Omega_{z 0}}, \\
\zeta_{3}=\left.\frac{d D_{T}^{12}}{d \Omega_{z}}\right|_{\Omega_{z}=\Omega_{z 0}}, \zeta_{4}=\left.\frac{d D_{T}^{22}}{d \Omega_{z}}\right|_{\Omega_{z}=\Omega_{z 0}},
\end{gathered}
$$

and are identical to those obtained using $f_{\omega_{z}}^{\prime}(\mathbf{e})$ in Hwang \& Pedley (2014).

We now eliminate $p^{\prime}$ in (2.15) following the standard procedure and rewrite $(2.15 a)$, $(2.15 b)$, and $(2.15 c)$ into a single equation in terms of $v^{\prime}$. We then consider a normal mode solution,

$$
v^{\prime}(x, y, t)=\hat{v}(y) e^{i(\alpha x-\omega t)}, n^{\prime}(x, y, t)=\hat{n}(y) e^{i(\alpha x-\omega t)},
$$

where $\alpha$ is the streamwise wavenumber and $\omega$ is the frequency. This leads to the following equations for linear stability:

$$
-i \omega\left(\begin{array}{cc}
\mathcal{M} & 0 \\
0 & 1
\end{array}\right)\left(\begin{array}{c}
\hat{v} \\
\hat{n}
\end{array}\right)+\left(\begin{array}{cc}
L_{O S} & i \alpha \operatorname{RiD} \\
L_{C}^{v} & L_{C}
\end{array}\right)\left(\begin{array}{c}
\hat{v} \\
\hat{n}
\end{array}\right)=0
$$

where

$$
\begin{gathered}
\mathcal{M}=\alpha^{2}-\mathcal{D}^{2} \\
L_{O S}=i \alpha U_{0} \mathcal{M}+i \alpha \mathcal{D}^{2} U_{0}+\frac{1}{R e} \mathcal{M}^{2} \\
L_{C}^{v}=\mathcal{D} n_{0}+G_{1}\left[-\zeta_{1} n_{0} \mathcal{M}+\frac{i}{\alpha} \mathcal{D}\left(\zeta_{2} n_{0} \mathcal{M}\right)\right] \\
-G_{2}\left[-\zeta_{3} \mathcal{D} n_{0} \mathcal{M}+\frac{i}{\alpha} \mathcal{D}\left(\zeta_{4} \mathcal{D} n_{0} \mathcal{M}\right)\right] \\
L_{C}=i \alpha U_{0}+i \alpha\left\langle e_{1}\right\rangle_{0}+\left\langle e_{2}\right\rangle_{0} \mathcal{D}+\mathcal{D}\left\langle e_{2}\right\rangle_{0} \\
+\frac{1}{\operatorname{ScRe}}\left[\alpha^{2} D_{T 0}^{11}-2 i \alpha D_{T 0}^{12} \mathcal{D}-D_{T 0}^{22} \mathcal{D}^{2}-i \alpha \mathcal{D} D_{T 0}^{12}-\mathcal{D} D_{T 0}^{22} \mathcal{D}\right]
\end{gathered}
$$

with boundary conditions

$$
\begin{gathered}
\left.\hat{v}\right|_{y= \pm 1}=\left.\mathcal{D} \hat{v}\right|_{y= \pm 1}=0 \\
{\left.\left[\left\langle e_{2}\right\rangle_{0} \hat{n}-\frac{1}{\operatorname{ScRe}}\left(i \alpha D_{T 0}^{12}+D_{T 0}^{22} \mathcal{D}\right) \hat{n}+\frac{i}{\alpha}\left(G_{1} \zeta_{2} n_{0}-G_{2} \zeta_{4} \mathcal{D} n_{0}\right) \mathcal{M} \hat{v}\right]\right|_{y= \pm 1}=0 .}
\end{gathered}
$$

Here, $\mathcal{D} \equiv d / d y, L_{O S}$ is the Orr-Sommerfeld operator, $L_{C}^{v}$ the coupling operator between $\hat{v}$ and $\hat{n}$ in the equation for $\hat{n}$, and $L_{C}$ the homogeneous part of the equation for $\hat{n}$, 
respectively. The parameters $G_{1}$ and $G_{2}$ in $(2.21 d)$ are defined as

$$
G_{1}=\frac{1}{D_{R}}=\frac{V_{c}^{*}}{D_{R}^{*} h}, G_{2}=\frac{1}{\operatorname{ScRe} D_{R}}=\frac{V_{c}^{* 2} \tau}{D_{R}^{*} h^{2}} .
$$

As discussed in Hwang \& Pedley (2014), $G_{1}$ indicates the importance of swimming relative to rotational diffusion and $G_{2}$ represents the importance of translational diffusion relative to rotational diffusion. Finally, it should be pointed out that $L_{C}$ is not a simple advection-diffusion operator because of the term $\mathcal{D}\left\langle e_{2}\right\rangle_{0}$ in the top line of $(2.21 e)$. This term appears to represent the production caused by the gradient in the mean swimming vector field $\mathcal{D}\left\langle e_{2}\right\rangle_{0}$, and it is found to play a crucial role in generating the instabilities in the present study as we shall see in $§ 4.2$.

\subsection{Numerical methods}

The equations for the basic state (2.7) are solved numerically. The horizontal direction is discretized using a Chebyshev-collocation method (Weideman \& Reddy 2000), and the boundary conditions are implicitly imposed on the discretized operator so that its inversion is numerically possible. The steady solution of (2.7) is then obtained using the Newton-Raphson iteration with a prescribed flow rate $Q$. For the smallest $Q$ considered, the iteration is started with a guess $U_{0}(y)=3 Q / 4\left(y^{2}-1\right)$ and $n_{0}(y)=1$. On gradually increasing $Q$, the initial guess is obtained using a numerical continuation: the solution obtained for the previous $Q$ is imposed as the initial guess for the new $Q$. The numerical solution is obtained with $N_{y}=151$, and it is found to be identical to that with $N_{y}=251$ within the residual of the iteration. The solution is also validated by an independently written numerical solver of (2.7). In this solver, the horizontal direction is discretized using a second-order finite volume method, and the solution is obtained by solving the unsteady (2.7) for which the time integration is conducted semi-implicitly: the diffusion terms are marched using the second-order Crank-Nicolson method, and the rest of the terms are advanced using a third-order Runge-Kutta method. The solution is obtained with $N_{y}=501$ to capture the spectral accuracy of the Newton-Raphson solver, and shows excellent agreement with that of the Newton-Raphson solver.

The equations for linear stability $(2.21)$ are solved by modifying the solver validated in our previous study (Hwang \& Pedley 2014). The horizontal direction is discretised using the same Chebyshev-collocation method. The discretized eigenvalue problem is solved using the function eig in Matlab. The computation is performed with $N_{y}=151$, showing no difference from the results with $N_{y}=251$.

\subsection{Parameters}

Table 1 summarises a list of the parameters and their values in the present study. The values of the parameters are taken from those for $C$. nivalis as in previous studies (e.g. Pedley \& Kessler 1990; Bees \& Hill 1998; Pedley 2010b; Hwang \& Pedley 2014). The width of the channel is chosen to be close to the scale of the laboratory experiments (Kessler 1985a, 1986; Denissenko \& Lukaschuk 2007). It should be pointed out that the mean cell number densities $N$ considered is one order of magnitude lower than that in the typical experimental condition. However, the onset of the instability is, in fact, found under such conditions at least within the framework of the present model. We note that this does not yield any inconsistency with the existing experiments as the critical cell number density for the blip instability has not been discussed at all. Furthermore, performing a linear stability analysis with the $N$ used in the experiment would not be physically so meaningful from a theoretical perspective as this regime is quite far from 


\begin{tabular}{lccc}
\hline Parameter & Description & Value (Ref. value) & Units \\
$\rho$ & Fluid density & 1 & $\mathrm{~g} / \mathrm{cm}^{3}$ \\
$g$ & Gravitational acceleration & 980 & $\mathrm{~cm} / \mathrm{s}^{2}$ \\
$\nu$ & Kinematic viscosity & 0.01 & $\mathrm{~cm}^{2} / \mathrm{s}$ \\
$d(=2 h)$ & Channel width & $0.4 \sim 0.8(0.4)$ & $\mathrm{cm}$ \\
$N$ & Cell mean number density & $0 \sim 2 \times 10^{5}$ & $\mathrm{cells} / \mathrm{cm}^{3}$ \\
$\Delta \rho / \rho$ & Relative cell density & 0.05 & - \\
$v$ & Cell volume & $2.1 \times 10^{-9}$ & $\mathrm{~cm}^{3}$ \\
$g^{\prime}(=g \Delta \rho / \rho)$ & Relative gravity & 49 & $\mathrm{~cm} / \mathrm{s}^{2}$ \\
$B$ & gyrotactic time scale & 3.4 & $\mathrm{sec}$ \\
$V_{c}^{*}$ & Swimming speed & $6.3 \times 10^{-3}$ & $\mathrm{~cm} / \mathrm{s}$ \\
$\tau$ & Correlation time scale & $2 \sim 5(5)$ & $\mathrm{s}$ \\
$D_{V}^{*}\left(=V_{c}^{* 2} \tau\right)$ & Nominal translation cell diffusivity & $1.98 \times 10^{-4}$ & $\mathrm{~cm} / \mathrm{s}$ \\
$D_{R}^{*}$ & Rotational diffusivity & $0.067 \sim 0.147(0.067)$ & $1 / \mathrm{s}$
\end{tabular}

TABLE 1. Parameters and their values in the present study (the ones in parentheses indicate the reference values). The parameters for the cell properties are taken from the data for C. nivalis (Pedley \& Kessler 1990; Bees \& Hill 1998; Pedley 2010b; Hwang \& Pedley 2014).

\begin{tabular}{|c|c|c|}
\hline Parameter & Description & Value (Ref. value) \\
\hline $\mathrm{Sc}$ & Schmidt number & $50 \sim 126(50)$ \\
\hline $\mathrm{Ri}$ & Richardson number & $0 \sim 200$ \\
\hline$Q$ & Dimensionless flow rate & $0 \sim 80$ \\
\hline Re & Reynolds number based on $V_{c}^{*}$ & $0.13 \sim 0.25(0.13)$ \\
\hline $\operatorname{Re}_{b}$ & Reynolds number based on the bulk flow velocity & $0 \sim 10$ \\
\hline$G_{1}\left(=1 / D_{R}\right)$ & see $(2.21 h)$ & $0.21 \sim 0.47(0.47)$ \\
\hline$G_{2}$ & see $(2.21 h)$ & $0.018 \sim 0.074(0.074)$ \\
\hline$\lambda$ & inverse of gyrotactic time scale normalised by $D_{R}^{*}$ & $1 \sim 2.2(2.2)$ \\
\hline$D_{R}$ & Rotational diffusivity normalised by $V_{c}^{*} / h$ & $2.1 \sim 4.7(2.1)$ \\
\hline
\end{tabular}

TABLE 2. Dimensionless parameters and their values in the present study (the ones of parentheses indicate the reference values).

the onset of instability. Based on the parameter values given in table 1 , the dimensionless parameters and their values are obtained as in table 2 .

\section{Results}

\subsection{Basic state}

The basic state is first calculated by solving (2.7), and is reported in figure 4 for a sufficiently large $\operatorname{Ri}(=90)$. For zero flow rate $(Q=0), U_{0}(y)=0$ and $n_{0}(y)=1$ as discussed. These solutions continuously deform as the flow rate $Q$ is gradually increased. For small flow rates $(Q<2)$, the velocity profile exhibits upward backflow near the wall $(|y|>0.6)$ though the bulk fluid flows downward (figure $4 a$ ). This backflow typically appears at large $\mathrm{Ri}$ as will be discussed with figure 5 . The cell-number density profile $n_{0}(y)$ shows its maximum at the channel centre $y=0$ (figure $4 b$ ), and it is clearly a consequence of the gyrotaxis of the cells as in the experiment of Kessler (1985a): the downward flow yields the cells to swim towards the centreline (i.e. $\left\langle e_{1}\right\rangle_{0}>0$ at $y<0$ with $\Omega_{z 0} \geqslant 0$ and $\left\langle e_{1}\right\rangle_{0}<0$ at $y>0$ with $\left.\Omega_{z 0} \leqslant 0\right)$ while they swim upward $\left(\left\langle e_{2}\right\rangle_{0}>0\right)$. In this respect, it is interesting to note that $n_{0}(y)$ at the walls $(y= \pm 1)$ in this case is 

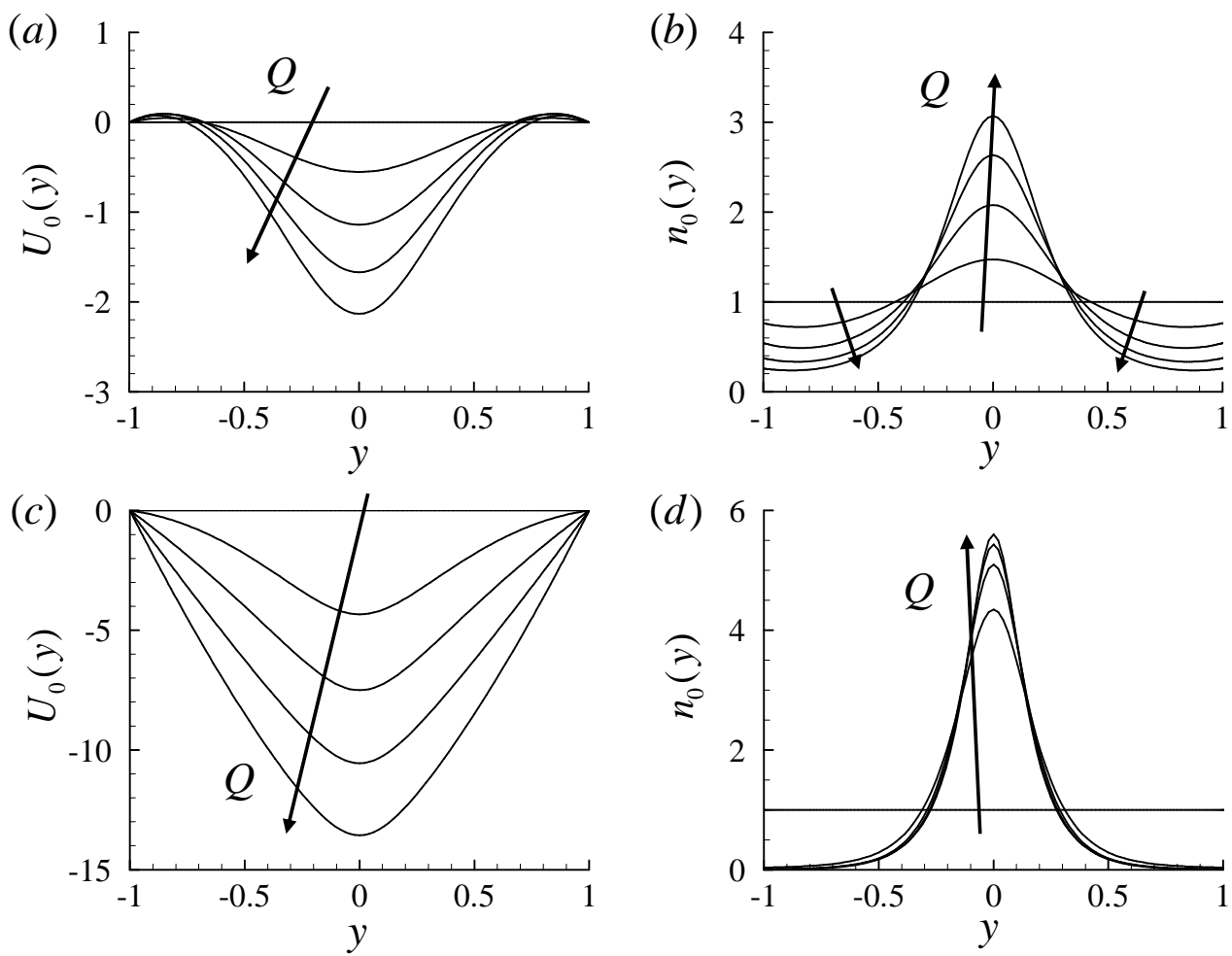

Figure 4. Profiles of the basic state respectively for $(a, b)$ low $(Q=0,0.4,0.8,1.2,1.6)$ and $(c, d)$ high flow rates $(Q=0,5,10,15,20)$ at $\mathrm{Ri}=90:(a, c)$ vertical velocity $U_{0}(y) ;(b, d)$ cell-number density $n_{0}(y)$.

slightly larger than it is close by due to the upward backflow. As the flow rate $Q$ is further increased, the upward backflow in $U_{0}(y)$ diminishes due to the strong pressure gradient applied downward (figure $1 c$ ). Also, $U_{0}(y)$ becomes closer to the parabolic profile given in (2.10a) because the forcing by the pressure gradient dominates over the gravitational one. The peak value of the cell-number density profile $n_{0}(y)$ at the channel centre is found to increase with the flow rate (figure $4 d$ ), indicating that the beam-like structure along the channel centre is more focused on increasing $Q$ at least for the values considered here. However, it should be mentioned that this behaviour may not persist at very large flow rates. If the flow rate $Q$ is very large, local $\Omega_{z 0}$ would also be very large. In this case, $\left\langle e_{2}\right\rangle_{0}$ decreases with the increase of $Q$ while $D_{T 0}^{22}$ approaches a constant value $D_{T 0}^{22} \simeq 0.33$ (see figure 2). Since $n_{0}(y)$ is given by (2.10b) in the limit of $Q \rightarrow \infty$, the width of the focussing in $n_{0}(y)$ at the channel centre (i.e. $l \sim D_{T 0}^{22} /\left\langle e_{2}\right\rangle_{0}$ ) would become thicker with an increase of $Q$. However, we should note that this could be an artifact of the present model as the expression for the diffusivity tensor (2.3) would not be reliable at very large $\Omega_{z 0}$ (or $Q$ ). For further detailed discussion on this issue, the reader may refer to appendix in Hwang \& Pedley (2014) where the relevance of the expression (2.3) is analysed in comparison to the generalised Taylor dispersion theory.

The range of the flow rate $Q$ and the Richardson number Ri leading to the backflow in $U_{0}(y)$ is also identified by further inspecting (2.9). Integrating (2.9) over $y \in[-1,0]$ 


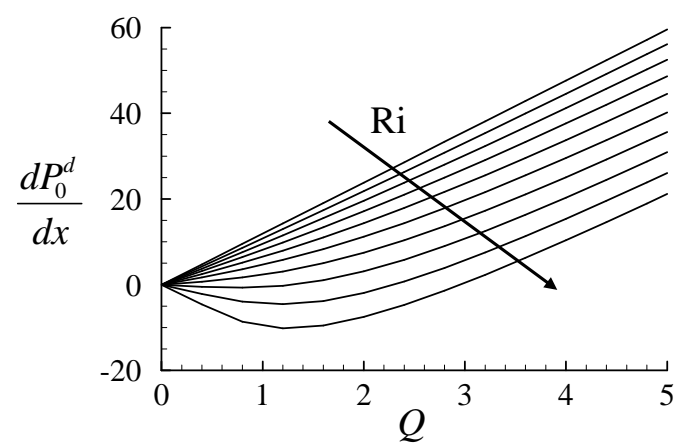

FiguRE 5. Dependence of the relation between the driving pressure and the flow rate on the Richardson number $(\mathrm{Ri}=0,10,20,30, \ldots, 90)$.

gives

$$
\frac{d P_{0}^{d}}{d x}=-\left.\frac{1}{\operatorname{Re}} \frac{d U_{0}}{d y}\right|_{y=-1} .
$$

If the backflow in $U_{0}(y)$ appears near the wall, $d U_{0} /\left.d y\right|_{y=-1}>0$, yielding $d P_{0}^{d} / d x<0$. Figure 5 shows the relation between $d P_{0}^{d} / d x$ and $Q$ obtained from the computed basic state for a number of Ri. The region for $d P_{0}^{d} / d x<0$ appears at relatively small $Q(<3)$ and large $\operatorname{Ri}(>70)$, indicating that the the backflow appears if the gravitational forcing dominates over the pressure gradient in the flow-field formation. Furthermore, $d P_{0}^{d} / d x<$ 0 implies that the driving pressure is applied upward, suggesting that the backflow is caused by an upward pressure gradient. Therefore, it appears that the downward flow in this case is generated essentially by the gravitational force, and the role of the imposed pressure gradient is limited to simply balancing with the gravitational force so that the given flow rate is maintained. It should be pointed out that this feature would make it difficult to interpret an analysis like the present one but with $d P_{0}^{d} / d x$ prescribed instead of flow rate $Q$. As shown in figure 5 , for a given $d P_{0}^{d} / d x$, two steady solutions emerge at sufficiently large Ri. A time dependent simulation using the numerical solver used for validation shows that the one with smaller $Q$ is an unstable steady solution. This suggests that if $d P_{0}^{d} / d x$ is chosen as the control parameter for the flow rate, the steady solutions exhibit a saddle-node bifurcation on increasing $d P_{0}^{d} / d x$, creating an additional complexity in analyzing the flow field.

\subsection{Linear stability analysis}

Now, we perform a linear stability analysis with the reference parameters given in tables 1 and 2. We first consider a uniform suspension without any flow (i.e. $n_{0}(y)=1$ and $Q=0)$. Figure 6 shows the growth rate of the two most unstable modes with respect to the vertical wavenumber $\alpha$ for several $\mathrm{Ri}$ near the onset of the instability, $\mathrm{Ri}_{c} \simeq 66$ (note that $\mathrm{Ri}=50$ corresponds to $N \simeq 1 \times 10^{5}$ cells $/ \mathrm{cm}^{3}$ for the reference parameters). It is found that one branch depends on $\mathrm{Ri}$ (figure $6 a$ ), whereas the other remains unchanged regardless of the value of $\mathrm{Ri}$ (figure $6 b$ ). While the former gives a non-trivial eigenfunction, the latter is numerically found to yield a simple eigenfunction, $\hat{v}=0$ and $\hat{n}=$ const. This feature in the latter enables us to analytically obtain its dispersion relation as $\omega=\alpha\left\langle e_{1}\right\rangle_{0}-i D_{T 0}^{11} \alpha^{2} /(\mathrm{ScRe})$, and this is indeed identical to the numerical one given in figure $6(b)$. We note that this mode gives zero growth rate at $\alpha=0$ (i.e. $\omega_{i}=0$ ). However, the constraint given in (2.14) would exclude any initial condition for (2.15) that would give rise to this mode, so it can be safely neglected. 

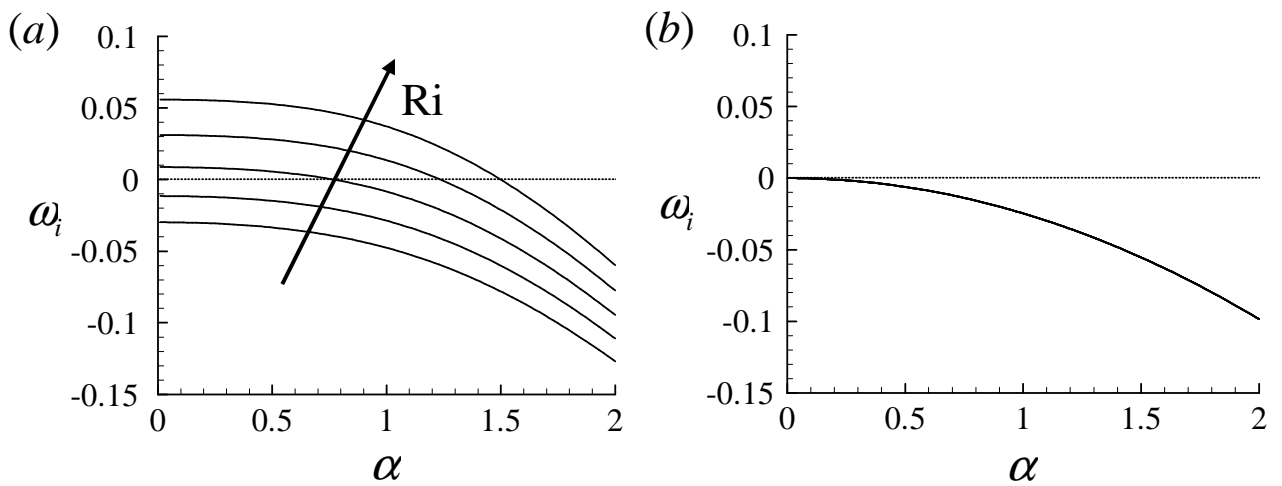

FiguRE 6 . Growth rate of the first two most unstable modes in stationary suspensions $(Q=0)$ with the vertical wavenumber $\alpha$ near the critical $\mathrm{Ri}_{c} \simeq 66(\mathrm{Ri}=50,60,70,80,90):(a)$ the mode depending on $\mathrm{Ri}$ and $(b)$ the one independent of $\mathrm{Ri}$.
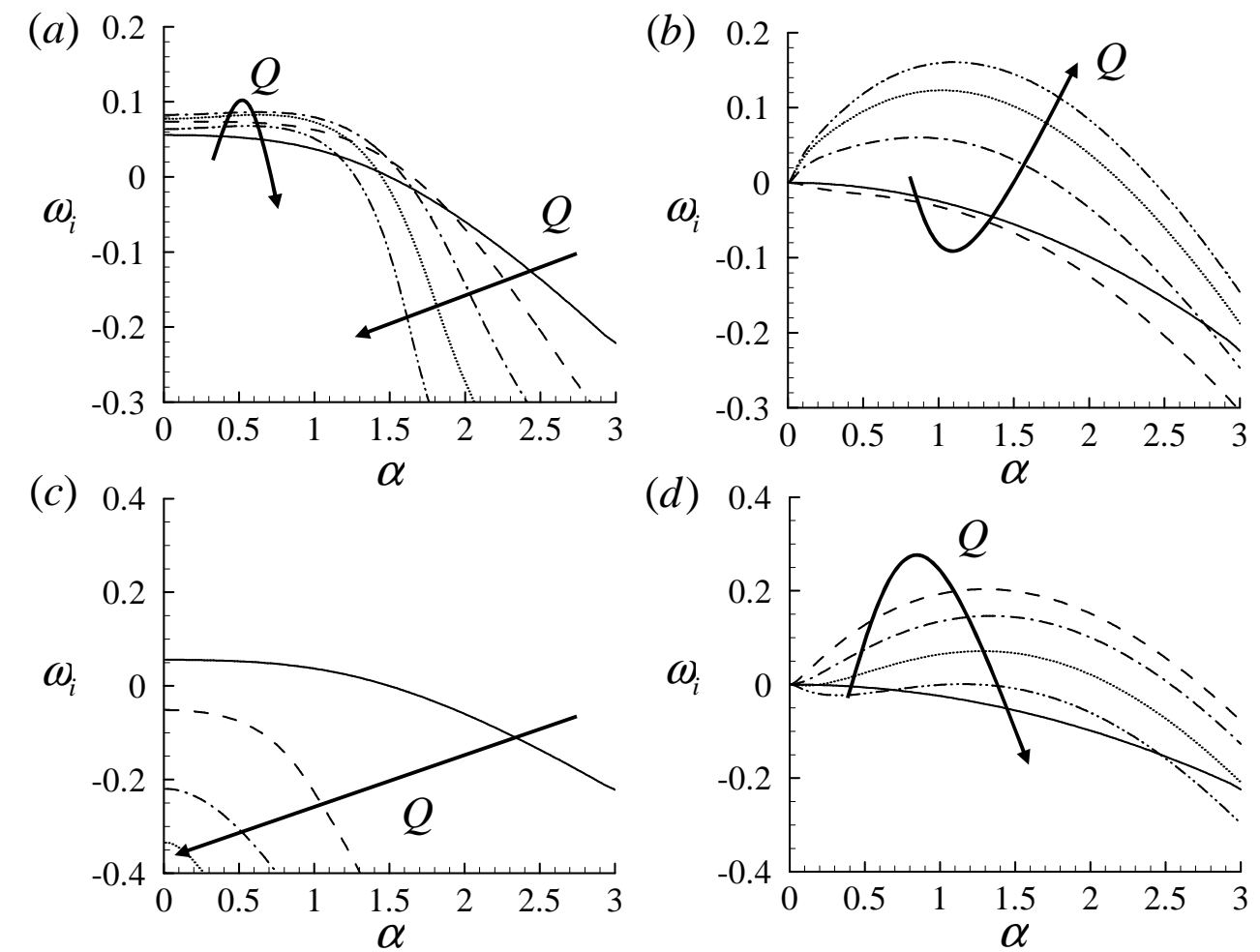

FiguRE 7. Growth rate with the vertical wavenumber $\alpha$ for $(a, b)$ small $(Q=0,0.4,0.8,1.2,1.6)$ and $(c, d)$ large $(Q=0,4,8,12,16)$ flow rates $(\mathrm{Ri}=90):(a, c)$ sinuous and $(b, d)$ varicose modes. In $(a, b),-, Q=0 ;---, Q=0.4 ;-\cdots, Q=0.8 ; \cdots \cdots \cdots, Q=1.2 ; \cdots \cdots, Q, Q=1.6$, and, in $(c, d), \stackrel{\longrightarrow}{\longrightarrow} Q=0 ;-\cdots, Q=4 ;-\cdot--, Q=8 ; \cdots \cdots \cdots, Q, Q=12 ; \cdots-\cdots, Q, Q=16$.

On increasing the flow rate $Q$, the branches of the two modes continuously change as shown in figure 7 . The mode depending on $\mathrm{Ri}$ at $Q=0$ develops into the sinuous mode (see also figure $8 a$ ), while the one independent of $\mathrm{Ri}$ at $Q=0$ smoothly changes into the varicose mode (see also figure $8 b$ ). For small flow rates $(Q<1)$, the sinuous mode at low vertical wavenumbers $(\alpha<1)$ is slightly destabilised with a small increase of $Q$ 
(a)

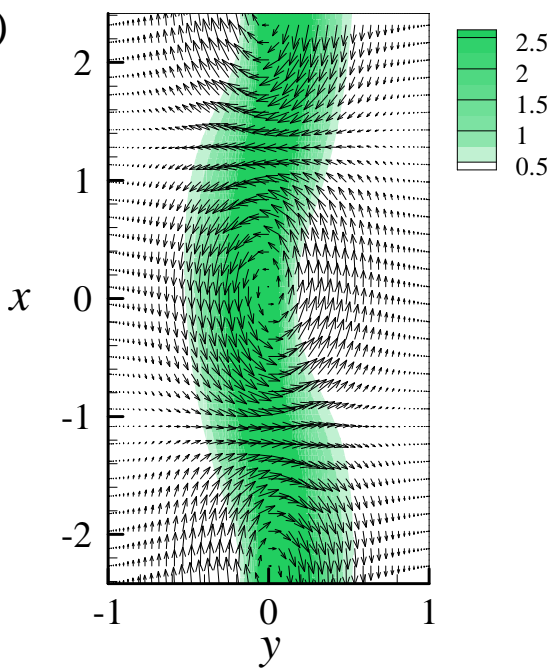

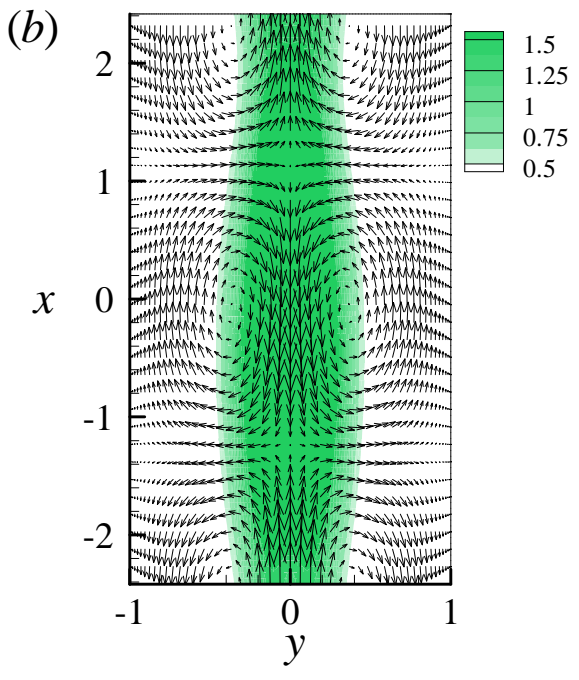

FigURE 8. Spatial structure of the $(a)$ sinuous and $(b)$ varicose modes $(R i=90, Q=4$, and $\alpha=1.3)$. Here, the contours indicate $n_{0}(y)+\gamma n^{\prime}(y, x)$ with an arbitrary value of $\gamma$ for visualization, and the vectors represent the vertical and horizontal perturbation velocity field.

(figure $7 a)$ whereas at high wavenumbers $(\alpha>1.5)$ it is rapidly stabilised. However, as $Q$ is further increased, the sinuous mode for all $\alpha$ is stabilised, and this tendency continues even at very large flow rates (figure $7 c$ ). On the other hand, the varicose mode is significantly destabilised with an increase of $Q$ except at very small $Q(<0.4)$ (figure $7 b)$. It becomes the most unstable mode for most of the flow rates considered $(Q>2$ in this case). The destabilization with the increase of $Q$ continues until $Q \simeq 4 \sim 5$, after which the varicose mode turns out to be slowly restabilised (figure $7 d$ ) on increasing $Q$. The varicose mode also becomes stable at very large flow rates $(Q>16)$.

The typical spatial structure of the sinuous and the varicose modes is visualised in figure 8 . The sinuous mode is characterised by a meandering motion of the focused beam along with an alternating column of vortices, spanning the whole width of the channel (figure $8 a$ ). The rotational direction of the vortex alternates along the vertical direction, and the focussed beam of the cell concentration meanders along the downwash side of each vortex. On the other hand, the beam-like structure in the varicose mode exhibits a periodic thickness variation along the vertical direction and a pair of counter-rotating vortices are aligned along the beam (figure $8 b$ ). This structure is clearly reminiscent of the blip instability observed in the downward pipe flow experiment of Kessler $(1985 a, 1986)$, and many other features of the varicose mode are also found to be strikingly similar to those of the blip instability as we shall see further in $\S 4.1$.

In figure 9 , we show contours of the largest growth rate $\omega_{i, \max }$ and the corresponding $\alpha_{\max }$ in the $Q$-Ri plane. Here, the values of $\alpha$ used to search $\alpha_{\max }$ are chosen to vary from $\alpha=0.01$ so that the branch giving $\omega_{i}=0$ at $\alpha=0$ is not numerically included (figure $6 b)$. However, even in this case, drawing a smooth neutral stability curve $\left(\omega_{i}=0\right)$ on the contour has been found to be difficult because this branch still gives $\omega_{i}$ of the most unstable mode to be close to zero at the smallest $\alpha(=0.01)$. For this reason, we simply provide a guide isoline $\omega_{i}=0.001$ (the solid line in figure $9 a$ ), which appears to be a little smoother than the precise neutral line $\omega_{i}=0$. For $Q=0$, the instability appears at $\mathrm{Ri} \geqslant \mathrm{Ri}_{c}(\simeq 66)$. As discussed above, the mode associated with this instability transits into the sinuous mode on increasing $Q$, and is rapidly stabilised for $Q>0.4$ (see also figures 

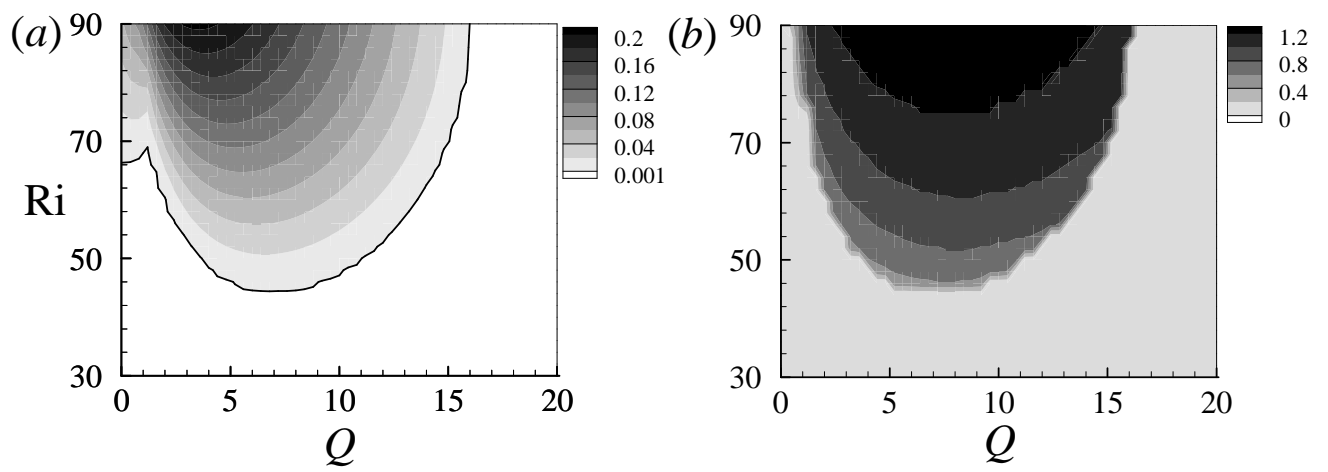

Figure 9. (a) The maximum growth rate $\omega_{i, \max }$ and $(b)$ the corresponding $\alpha_{\max }$ in the Ri- $Q$ plane. Here, $\alpha$ is chosen from $\alpha=0.01$ to $\alpha=3.01$ with an increment $\Delta \alpha=0.02$. The solid line in $(a)$ is $\omega_{i}=0.001$.

$7 a$ and $c$ ). On the other hand, the varicose mode is significantly destabilised on increasing $Q$, yielding a branch switching behaviour with a kink near $Q \simeq 1.5$ in the contour of $\omega_{i, \max }$ (figure $9 a$ ). The varicose mode gives the largest growth rate $\omega_{i, \max }$ for $Q \gtrsim 1.5$. For sufficiently large flow rates $(Q \gtrsim 5)$, the varicose mode is stabilised as the flow rate is increased. The most unstable varicose mode involves a non-zero $\alpha_{\max }(\simeq 0.5 \sim 1.2)$ as already discussed (figure $9 b$ ). The vertical wavenumber giving the largest growth rate is found to increase on increasing $Q$ for $Q \lesssim 8$, and it then decreases with a further increase of $Q(\gtrsim 8)$.

\subsection{Other parameters}

In this section, we test the robustness of the present results by examining a few different sets of the parameters. As discussed previously, a limitation of the present model is the lack of an experimentally justified expression for the translational diffusivity tensor. We therefore focus on investigating how the results with the reference parameters would change as the diffusion characteristics of the system are changed. The following three parameters are considered to vary: 1) $\tau$ the correlation time scale in the translational diffusivity model $(2.3) ; 2) h$ the half width of the channel; 3) $D_{R}^{*}$ the rotational diffusivity. First, varying $\tau$ only changes the Schmid number Sc while the rest of the dimensionless parameters remain the same. This therefore allows us to examine solely the role of the translational diffusivity. Second, varying the half width of the channel $h$, which also enables us to test the robustness to the flow geometry, changes Re, Ri, and $D_{R}$ in (2.5). However, the change in $D_{R}$ does not yield a change in the solution of $(2.5 d)$ because $\boldsymbol{\Omega}$ in it is also made dimensionless with the length scale $h$. Since Ri is also a control parameter for the instabilities, varying $h$ would only change Re. Finally, varying the rotational diffusivity yields a change of $\lambda\left(=1 /\left(2 B D_{R}^{*}\right)\right)$ in $(2.5 d)$. This therefore results in a change in the statistical properties such as $\langle\mathbf{e}\rangle$ and $\mathbf{D}_{T}$, leading the entire system to experience an intricate change. However, it is worth pointing out that the increase of $D_{R}^{*}$ generally weakens the role of the swimming in the system: for example, in the limit of $D_{R}^{*} \rightarrow \infty,\langle\mathbf{e}\rangle=0$ and $\mathbf{D}_{T}=1 / 3 \mathbf{I}$ in (2.5c), indicating that the effect of the gyrotaxis would diminish.

Figure 10 shows the contours of $\omega_{i, \max }$ and $\alpha_{\max }$ in the $Q$-Ri plane for $\tau=2 \mathrm{~s}$ ( $\mathrm{Sc}=126)$. The overall tendency of the contours is qualitatively similar to that for the reference value, and only a quantitative change appears. For all flow rates $Q$, the instability appears at much lower $R i$ (figure 10a) compared to that with $\tau=5 \mathrm{~s}$ as 

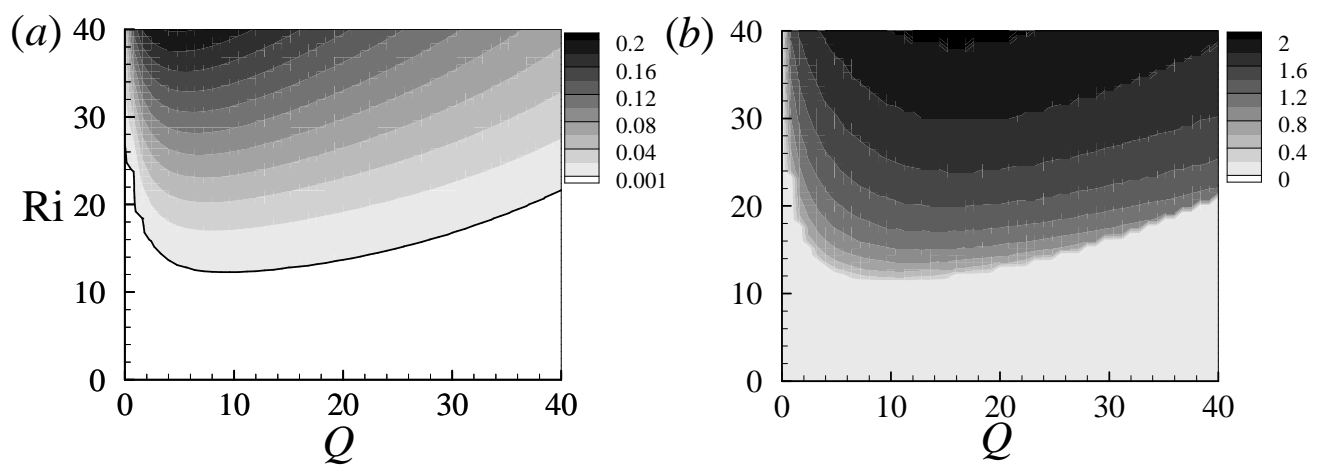

Figure 10. Contours of $(a)$ the maximum growth rate $\omega_{i, \max }$ and $(b)$ the corresponding $\alpha_{\max }$ in the $\operatorname{Ri}-Q$ plane for $\tau=2 \mathrm{~s}(\mathrm{Sc}=126)$. Here, $\alpha$ is chosen from $\alpha=0.01$ to $\alpha=3.01$ with an increment $\Delta \alpha=0.02$. The solid line in $(a)$ is $\omega_{i}=0.001$.
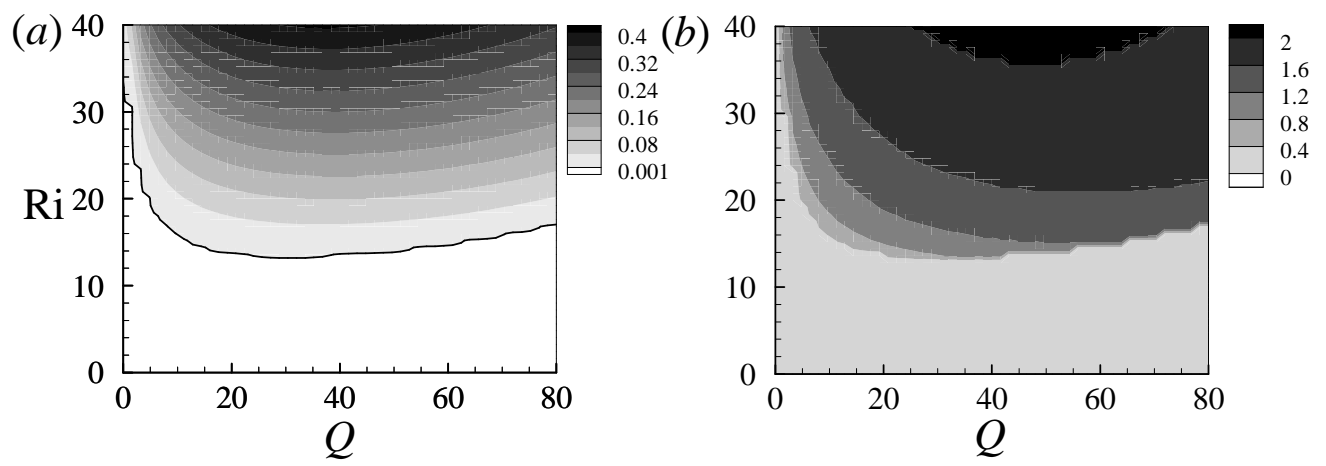

FIgURE 11. Contours of $(a)$ the maximum growth rate $\omega_{i, \max }$ and $(b)$ the corresponding $\alpha_{\max }$ in the $\mathrm{Ri}-Q$ plane for $h=0.4 \mathrm{~cm}(R e=0.25)$. Here, $\alpha$ is chosen from $\alpha=0.01$ to $\alpha=3.01$ with an increment $\Delta \alpha=0.02$. The solid line in $(a)$ is $\omega_{i}=0.001$.

expected (figure $9 a$ ). With a small increase of the flow rate $(Q<5)$, the varicose mode is destabilised as in the case of $\tau=5 \mathrm{~s}$ (figure 10a), and the corresponding $\alpha_{\max }$ is augmented. Given the fact that the overall translational diffusivity of the cell-number density is weaker, such a change would not be surprising. It is also interesting to note that the varicose instability in this case is persistent even at fairly high flow rates $(Q=40)$ although its growth rate tends to decay on increasing $Q$.

The effect of an increase of the half width of the channel $(h=0.4 \mathrm{~cm})$ is shown in figure 11. This change yields a decrease of the dimensionless fluid viscosity and the translational diffusivity of the cell-number density as the Reynolds number increases $(\mathrm{Re}=0.13 \rightarrow$ 0.25 ). We also note that, in this case, $\mathrm{Ri} \simeq 100$ for $N \simeq 1 \times 10^{5} \mathrm{cells} / \mathrm{cm}^{3}$. Not surprisingly, the overall change caused by the increase of the channel width is qualitatively similar to that for $\tau=2 \mathrm{~s}$ (figure 10): the critical Ri for the onset of the instability are considerably lowered at all $Q$, and the $\alpha_{\max }$ is also increased. The varicose mode in this case is even more persistent on increasing $Q$, and it is not completely restabilised even at $Q=80$ for the considered Ri.

Finally, the dependence on the rotational diffusivity is tested with $D_{R}^{*}=0.147 \mathrm{~s}^{-1}$ $(\lambda=1)$, a value significantly larger than the reference $D_{R}^{*}\left(=0.067 \mathrm{~s}^{-1}\right)(\lambda=2.2)$, and is shown in figure 12. The much higher values of $\operatorname{Ri}_{c}(>250)$ at all the flow rates $Q$ than those in figure 9 suggest that the increase of $D_{R}^{*}$ has a stabilizing effect on the instability. 

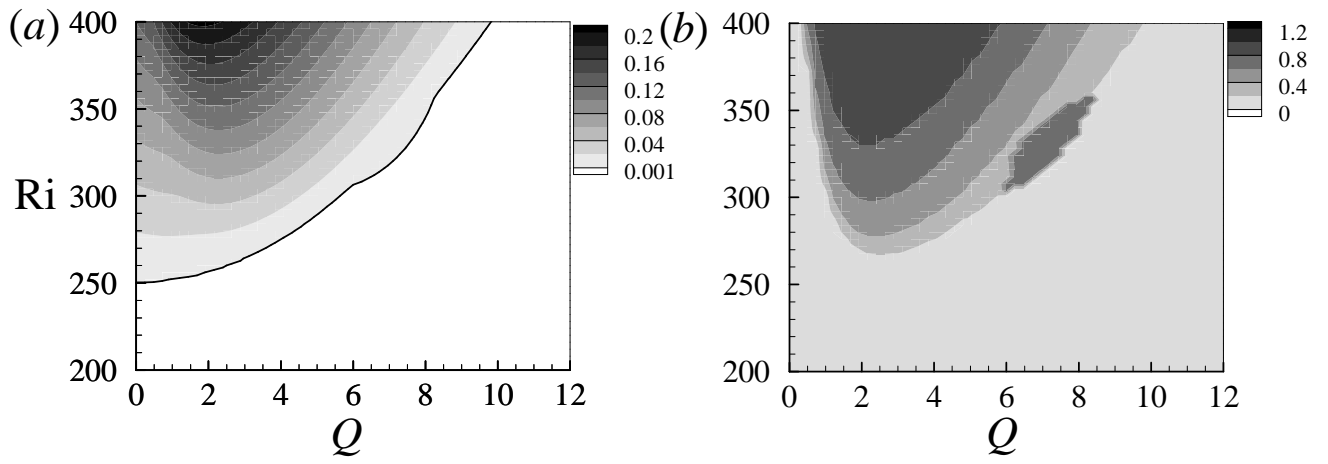

Figure 12. Contours of $(a)$ the maximum growth rate $\omega_{i, \max }$ and $(b)$ the corresponding $\alpha_{\max }$ in the $\mathrm{Ri}-Q$ plane for $D_{R}^{*}=0.147 \mathrm{~s}^{-1}(\lambda=1.0)$. Here, $\alpha$ is chosen from $\alpha=0.01$ to $\alpha=3.01$ with an increment $\Delta \alpha=0.02$. The solid line in $(a)$ is $\omega_{i}=0.001$.

Interestingly, the contours of $\omega_{i, \max }$ and $\alpha_{\max }$ in this case looks quite different from those in figures 9,10 , and 11. A closer scrutiny reveals that the most unstable mode is given by the sinuous mode instead of the varicose mode in most of the regions in the $\mathrm{Ri}-Q$ plane except for $\mathrm{Ri} \simeq 300 \sim 350$ and $Q \simeq 6 \sim 8$ where the varicose mode is found to be most unstable, resulting in a small island in the contours. This suggests that the increase of the rotational diffusivity stabilises the varicose mode more than the sinuous one and that the rotational diffusivity plays an important role in determining the competition dynamics between the sinuous and the varicose modes. However, the value considered in figure $12\left(D_{R}^{*}=0.147 \mathrm{~s}^{-1}\right)$ appears to be out of a biologically relevant range in the stationary condition: for instance, the experiment by Vladimirov et al. (2004) reported that the measured value of $\lambda$ for $C$. nivalis in a stationary medium varies in the range of $\lambda \simeq 1.8 \sim 3.0$. This suggests that the sinuous-mode dominance in the competition dynamics between the two modes would probably not be observed in reality.

\section{Discussion}

Thus far, we have analysed linear instabilities emerging in a downflowing suspension of gyrotactic microrganisms through a two-dimensional vertical channel. At zero flow rate $(Q=0)$, inspection of the two most unstable modes near the critical Richardson number $\mathrm{Ri}_{c}$ shows that one is destabilised by an increase of $\mathrm{Ri}$ while the other is found to be independent of Ri. On increasing the flow rate, the former continuously changes into the sinuous mode (figure $8 a$ ) whereas the latter is deformed into the varicose mode (figure $8 b$ ). For a given Ri (i.e. averaged cell concentration for a given flow configuration), the sinuous mode is slightly destabilised by a small increase of the flow rate and is quickly restabilised when it is further increased. On the other hand, the varicose mode is much more significantly destabilised than the sinuous one by an increase of the flow rate, being the most unstable mode in a wide range of the flow rate. At very large flow rates, both sinuous and varicose modes are found to be restabilised. These qualitative features are robust to changes of the parameters such as the translational diffusivity and the channel width as long as the value of the rotational diffusivity is kept in the biologically relevant range. 


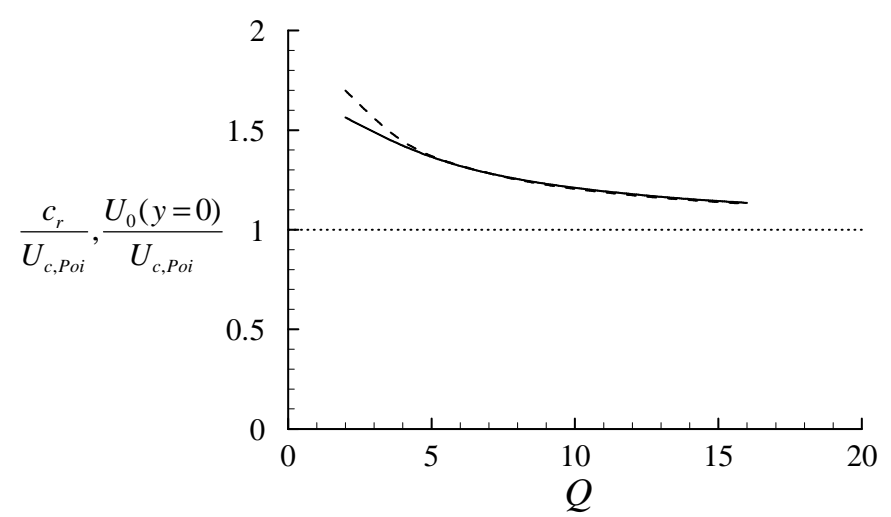

FIGURE 13. Centreline velocity $U_{0}(y=0)$ and phase speed of the varicose mode $c_{r}\left(=\omega_{r} / \alpha_{\max }\right)$ normalised by the corresponding centreline velocity of the plane Poiseulle flow $U_{c, P o i}(=3 Q / 4)$ with respect to the flow rate $Q(\mathrm{Ri}=90):-, c_{r} / U_{c, P o i} ;----, U_{0}(y=0) / U_{c, P o i}$.

\subsection{Comparison with experiments}

Let us first compare the present results with the original experiment by Kessler (1986) and the recent one by Denissenko \& Lukaschuk (2007). Although their flow configuration is composed of a vertical pipe rather than a channel as in the present study, a number of the present findings show qualitatively good agreement with those in the experiments. As mentioned previously, the spatial structure of the varicose mode is very similar to that of the blip instability: for example, the pair of counter rotating vortices in figure $8(b)$ is reminiscent of the vortex ring in the late stage of the blip instability in pipe flow (see e.g. figure 5 in Kessler 1986). The vertical wavenumber $\alpha_{\max }$ of the most unstable varicose mode in the present study has been robustly found to be $\alpha_{\max } \simeq 1 \sim 2$ regardless of the change of some parameters (figures 9,10 , and 11). This yields $\lambda_{x, \text { max }}^{*} \simeq 0.5 \sim 1.5 \mathrm{~cm}$, which compares fairly well with that observed in the experiments: e.g. $\lambda_{x}^{*} \simeq 1.5 \mathrm{~cm}$ in Kessler (1986) and $\lambda_{x}^{*} \simeq 0.5 \sim 0.7 \mathrm{~cm}$ in Denissenko \& Lukaschuk (2007). Furthermore, the experiments reported that the blips disappear at sufficiently high flow rates, and the same qualitative feature is retrieved as seen in figure 9.

The phase speed of the varicose mode is also compared with the falling speed of the blips. Figure 13 shows the phase speed of the varicose mode $c_{r}\left(=\omega_{r} / \alpha_{\max }\right)$ with the centreline velocity of the base flow $U_{0}(y=0)$. Here, both $c_{r}$ and $U_{0}(y=0)$ are normalised by $U_{c, P o i}$ to compare them with the centreline velocity of the plane Poiseulle flow at the same flow rate $U_{c, P o i}(=4 / 3 Q)$. The phase speed of the varicose mode $c_{r}$ is found to be faster than $U_{c, P o i}$, indicating that the varicose mode falls faster than the corresponding centreline velocity of the plane Poiseulle flow. Interestingly, it is almost identical to the centreline velocity of the base flow $U_{0}(y=0)$ except for $Q<4$ where $c_{r}$ is a little slower than $U_{0}(y=0)$, indicating that the varicose mode originates from $y=0$. Furthermore, this suggests that the faster speed of the varicose mode is essentially due to the focussed beam which increases the centreline flow speed via the gravitational force. This finding is consistent with the pipe flow experiment of Kessler (1986) where he reported that the blips fall faster than the corresponding centreline velocity of the Poiseulle flow $U_{c, P o i}$. The speed of the blips in his experiment was found as $2 \sim 5 U_{c, P o i}$, comparable with $c_{r}=1 \sim 2 U_{c, P o i}$ in figure 3 , given the flow geometry of the present study.

Despite such an encouraging comparison, care needs to be taken in interpreting the present results quantitatively. An important reason for this stems from the lack of a reliable model for the diffusivity tensor. As discussed, the rotational diffusivity is significantly 
augmented on increasing the local vorticity (or shear rate) according to a preliminary experimental observation (S. Furlan, T. J. Pedley \& R. E. Goldstein 2014, unpublished observation). This increase is found to continue until the local vorticity reaches the value at which the deterministic gyrotactic swimmer would begin to tumble. Since such an increase of the rotational diffusivity is found to significantly damp out the appearance of the varicose mode (figure 12), this would probably tend to stabilize the varicose mode at even smaller flow rates in reality. Furthermore, although the experiments were performed with dilute suspensions (typically less than $1 \%$ of the mean cell concentration), the local cell concentration in the region of the focussed beam could reach a quite high value. Therefore, at least in this region, the direct cell-cell interactions may not be negligible, and this would yield a quantitative difference from the experimental observation.

\subsection{Physical mechanisms of the instabilities: budget analysis}

Although a number of the present findings appear to be consistent with the experimental observations, it is not obvious what physical mechanisms are involved in such an encouraging comparison. This essentially stems from the fact that the instabilities in the present analysis could be associated with a number of different mechanisms, thus it is quite difficult to identify how such different physical mechanisms would cooperate and/or compete with each other. To overcome this difficulty in unveiling the underlying physical mechanisms, here we design a budget analysis for an instability which calculates the term-by-term contribution to its growth rate, similarly to the energy budget analysis given in turbulence literature (e.g Pope 2000). Before we formulate this analysis, it is helpful to sort out all the potential source terms of the instability. A careful inspection of the equations for linear stability (2.21) suggests that at least five different physical processes could be involved in generating the instabilities:

(a) Inflectional instability: The presence of inflection points in $U_{0}(y)$ (see figures $4 a$ and $c$ ) would be an instability mechanism from the well-known Rayleigh's theorem given in the inviscid limit (Drazin \& Reid 1981). Such inflection points in the velocity profile were also observed in the experiment of Kessler (1985a), and Pedley \& Kessler (1992) indeed speculated that they might play a role in generating the blip instability. From this viewpoint, it is interesting to note that the inflection points in $U_{0}(y)$ in the present study appear only at low flow rates where the varicose instability is significantly destabilised. We note that this mechanism essentially stems from the term $i \alpha \mathcal{D}^{2} U_{0} \hat{v}$ in $(2.21 c)$ (Drazin \& Reid 1981), and, in the absence of this term, the inflectional instability would not appear, as in plane Couette flow where the velocity is simply a linear function of the wall-normal coordinate.

(b) Gravity-driven instability: This mechanism is associated with the one observed in a dense falling line (or circular) plume through a surrounding light fluid. Considering the flow configuration of the present study, it would be relevant to refer to the work by Lister (1987) who examined linear stability of a dense line plume falling through a two-dimensional vertical channel. Using long vertical wavelength asymptotics, he found that only the varicose mode becomes unstable at low Reynolds numbers. The physical mechanism of the varicose mode essentially originates from the perturbation interacting with gravity at the interface of the plume and is related to a simple gravitational mechanism: i.e. if the plume is thicker than the mean thickness, it falls faster, whereas, if it is thinner, it falls more slowly. This mechanism appears to be associated with the term $\mathcal{D} n_{0} \hat{v}$ in $(2.21 d)$ which describes the interaction of a velocity perturbation with the gradient in the local cell-number density.

(c) Gyrotactic instability: The physical mechanism of the gyrotatic instability was discussed in the introduction as well as in a number of previous studies (Pedley et al. 
1988; Pedley \& Kessler 1990, 1992; Pedley 2010a; Hwang \& Pedley 2014). It is probable that this mechanism plays some role in generating the instabilities given the nature of the cells in the present study. This instability would originate from the terms multiplied by $G_{1}$ in $(2.21 d)$ (see also Hwang \& Pedley 2014) as $G_{1}$ characterises the importance of swimming relative to rotational diffusion. We note that if the cells do not swim, this term turns out to be zero.

(d) Diffusion-oriented instability: The instability driven by diffusion flux has not been well recognised until recently. In our recent study (Hwang \& Pedley 2014), we have shown that a local vortical perturbation creates a negative cross diffusion flux which drives the cells to move to locally denser regions. It should be pointed out that this feature arises because the cells are gyrotactic. The mechanism appears to be particularly important when the basic state of the cell concentration is unstably stratified as in shallow-layer bioconvection. Therefore, it is not obvious whether this mechanism would also play any role in generating the present instabilities. As discussed in Hwang \& Pedley (2014), this mechanism is associated with the terms multiplied by $G_{2}$ in $(2.21 d)$.

(e) Gradient in the cell swimming vector field: This last instability mechanism appears neither in uniform suspensions nor suspensions with a uniform shear. It appears only if the suspension is exposed to non-uniform shear (or vorticity) as in the present study. Although the role of this mechanism has not been discussed at all, the related term does appear e.g. in the equations of Ghorai \& Hill (1999). We note that this mechanism is represented by the term $\mathcal{D}\left\langle e_{2}\right\rangle_{0} \hat{n}$ in $(2.21 e)$, which describes production due to the gradient in the cell swimming vector field. As we shall see, this mechanism indeed plays a crucial role in generating the instabilities in the present study.

Now, we calculate the contribution of each of the terms discussed above to the instability found in the present study. We take a left multiplication of $(2.21 a)$ by a row vector $\left[\begin{array}{cc}\overline{\hat{v}} & \hat{n}^{-}\end{array}\right]$(the overbar indicates the complex conjugate) and integrate over the entire horizontal domain $\Omega_{y}=[-1,1]$. Taking the part contributing to the growth rate $\omega_{i}$ then yields

$$
\omega_{i}=E_{o s}+E_{v n}+E_{n v}+E_{n n}
$$

with

$$
\begin{aligned}
& E_{o s}=-\operatorname{Real}\left[\frac{\int_{\Omega_{y}} \overline{\hat{v}} L_{o s} \hat{v} d y}{\int_{\Omega_{y}} \alpha^{2}|\hat{v}|^{2}+|\mathcal{D} \hat{v}|^{2}+|\hat{n}|^{2} d y}\right], \\
& E_{v n}=\operatorname{Imag}\left[\frac{\int_{\Omega_{y}} \overline{\hat{v}} \alpha \operatorname{Ri} \mathcal{D} \hat{n} d y}{\int_{\Omega_{y}} \alpha^{2}|\hat{v}|^{2}+|\mathcal{D} \hat{v}|^{2}+|\hat{n}|^{2} d y}\right], \\
& E_{n v}=-\operatorname{Real}\left[\frac{\int_{\Omega_{y}} \overline{\hat{n}} L_{C}^{v} \hat{v} d y}{\int_{\Omega_{y}} \alpha^{2}|\hat{v}|^{2}+|\mathcal{D} \hat{v}|^{2}+|\hat{n}|^{2} d y}\right], \\
& E_{n n}=-\operatorname{Real}\left[\frac{\int_{\Omega_{y}} \overline{\hat{n}} L_{C} \hat{n} d y}{\int_{\Omega_{y}} \alpha^{2}|\hat{v}|^{2}+|\mathcal{D} \hat{v}|^{2}+|\hat{n}|^{2} d y}\right],
\end{aligned}
$$

where Real[·] and Imag[[] indicate the real and imaginary part of [·], respectively. Here, $E_{o s}, E_{v n}, E_{n v}$, and $E_{n n}$ are the contributions of $L_{o s}, i \alpha \operatorname{RiD}, L_{C}^{v}$, and $L_{C}$ in $(2.21 a)$, respectively. We also note that their denominators are positive definite. Based on this formula, it is possible to extract the contribution of the individual terms to the growth 


\begin{tabular}{|c|c|c|c|c|c|}
\hline Flow rate $Q$ & 0 & 4 & 8 & 12 & 16 \\
\hline$\omega_{i}$ & 0.02 & -0.40 & -0.75 & -1.10 & -1.43 \\
\hline$E_{o s}$ & -46.10 & -37.46 & -34.53 & -33.25 & -32.61 \\
\hline$E_{v n}$ & 46.11 & 37.33 & 34.31 & 32.96 & 32.24 \\
\hline$E_{n v}$ & 0.17 & 1.18 & 1.47 & 1.54 & 1.51 \\
\hline$E_{n n}$ & -0.16 & -1.44 & -2.00 & -2.35 & -2.58 \\
\hline$E_{n v}+E_{n n}$ & 0.01 & -0.26 & -0.53 & -0.81 & -1.07 \\
\hline$P_{i n f}$ & 0.00 & 0.00 & 0.00 & 0.00 & 0.00 \\
\hline$P_{\text {grav }}$ & 0.00 & -0.20 & -0.34 & -0.46 & -0.59 \\
\hline$P_{\text {gyro }}$ & 0.17 & 1.37 & 1.77 & 1.96 & 2.04 \\
\hline$P_{\text {diff }}$ & 0.00 & 0.02 & 0.04 & 0.05 & 0.06 \\
\hline$P_{e-\operatorname{grad}}$ & 0.00 & 0.44 & 0.62 & 0.69 & 0.71 \\
\hline
\end{tabular}

TABLE 3. Growth rate and the term-by-term contribution to the sinuous mode for the reference parameters $(\mathrm{Ri}=90$ and $\alpha=1.3)$. Here, the values leading to the largest contribution are denoted with the bold letters.

rate of the given instability: i.e.

$$
\begin{gathered}
P_{\text {inf }}=-\operatorname{Real}\left[\frac{\int_{\Omega_{y}} \overline{\hat{v}} i \alpha \mathcal{D}^{2} U_{0} \hat{v} d y}{\int_{\Omega_{y}} \alpha^{2}|\hat{v}|^{2}+|\mathcal{D} \hat{v}|^{2}+|\hat{n}|^{2} d y}\right], \\
P_{\text {grav }}=-\operatorname{Real}\left[\frac{\int_{\Omega_{y}} \overline{\hat{n}} \mathcal{D} n_{0} \hat{v} d y}{\int_{\Omega_{y}} \alpha^{2}|\hat{v}|^{2}+|\mathcal{D} \hat{v}|^{2}+|\hat{n}|^{2} d y}\right], \\
P_{\text {gyro }}=-\operatorname{Real}\left[\frac{\int_{\Omega_{y}} \overline{\hat{n}} G_{1}\left(-\zeta_{1} n_{0} \mathcal{M}+\frac{i}{\alpha} \mathcal{D}\left(\zeta_{2} n_{0} \mathcal{M}\right)\right) \hat{v} d y}{\int_{\Omega_{y}} \alpha^{2}|\hat{v}|^{2}+|\mathcal{D} \hat{v}|^{2}+|\hat{n}|^{2} d y}\right], \\
P_{\text {diff }}=\operatorname{Real}\left[\frac{\int_{\Omega_{y}} \overline{\hat{n}} G_{2}\left(-\zeta_{3} \mathcal{D} n_{0} \mathcal{M}+\frac{i}{\alpha} \mathcal{D}\left(\zeta_{4} \mathcal{D} n_{0} \mathcal{M}\right)\right) \hat{v} d y}{\int_{\Omega_{y}} \alpha^{2}|\hat{v}|^{2}+|\mathcal{D} \hat{v}|^{2}+|\hat{n}|^{2} d y}\right], \\
P_{e-\text { grad }}=-\operatorname{Real}\left[\frac{\int_{\Omega_{y}} \overline{\hat{n}} \mathcal{D}\left\langle e_{2}\right\rangle_{0} \hat{n} d y}{\int_{\Omega_{y}} \alpha^{2}|\hat{v}|^{2}+|\mathcal{D} \hat{v}|^{2}+|\hat{n}|^{2} d y}\right],
\end{gathered}
$$

where $P_{\text {inf }}, P_{\text {grav }}, P_{\text {gyro }}, P_{\text {diff }}$, and $P_{e-\text { grad }}$ represent the contribution of inflectional, gravitational, gyrotactic, diffusion-oriented, and cell-swimming-vector-field-gradient instability mechanisms, respectively.

Table 3 summarises how each term defined in (4.1) and (4.2) contributes to the growth rate of the sinuous mode. We first see the contribution of each operator: i.e. $E_{o s}, E_{v n}, E_{n v}$, and $E_{n n}$. In general, the absolute values of $E_{o s}$ and $E_{v n}$ from the momentum equation are larger than those of $E_{n v}$ and $E_{n n}$ from the equation for cell-number density. However, it appears that $E_{o s}$ and $E_{v n}$ are seen to be balanced with each other. Therefore, the quantity $E_{n v}+E_{n n}$ is seen to roughly give the growth rate $\omega_{i}$. This suggests that the sinuous mode essentially originates from the equation for cell-number density. We now take a look at the contribution of each instability production mechanism discussed above: i.e. $P_{\text {inf }}, P_{\text {grav }}$, $P_{\text {gyro }}, P_{d i f f}$, and $P_{e-\text { grad }}$. When the suspension is uniform $(Q=0)$, the contribution 


\begin{tabular}{lccccc} 
Flow rate $Q$ & 0 & 4 & 8 & 12 & 16 \\
\hline$\omega_{i}$ & -0.04 & 0.20 & 0.15 & 0.07 & -0.00 \\
\hline$E_{\text {os }}$ & 0.00 & -33.64 & -33.49 & -33.43 & -33.42 \\
$E_{\text {vn }}$ & 0.00 & 33.68 & 33.52 & 33.45 & 33.42 \\
$E_{n v}$ & 0.00 & 0.60 & 0.60 & 0.55 & 0.48 \\
$E_{n n}$ & -0.04 & -0.43 & -0.48 & -0.49 & -0.48 \\
\hline$E_{\text {vn }}+E_{n}$ & -0.04 & 0.17 & 0.12 & 0.06 & -0.00 \\
\hline$P_{\text {inf }}$ & & & & & \\
$P_{\text {grav }}$ & 0.00 & 0.00 & 0.00 & 0.00 & 0.00 \\
$P_{\text {gyro }}$ & 0.00 & 0.08 & 0.08 & 0.06 & 0.05 \\
$P_{\text {diff }}$ & 0.00 & 0.49 & 0.50 & 0.47 & 0.43 \\
$P_{\text {e-grad }}$ & 0.00 & 0.30 & 0.03 & 0.02 & 0.01 \\
\hline r & 0.00 & $\mathbf{1 . 1 3}$ & $\mathbf{1 . 5 0}$ & $\mathbf{1 . 7 3}$ & $\mathbf{1 . 9 0}$
\end{tabular}

TABLE 4. Growth rate and the term-by-term contribution term of the varicose mode for the reference parameters $(\mathrm{Ri}=90$ and $\alpha=1.3)$. Here, the values leading to the largest contribution are denoted with the bold letters.

to the mode comes only from the gyrotactic mechanism $\left(P_{\text {gyro }}\right)$. This confirms that the instability in the uniform suspension is the gyrotactic instability, consistent with previous studies (Pedley et al. 1988; Pedley \& Kessler 1990; Pedley 2010a). For Q > 0 , the gyrotatic mechanism still yields the largest contribution to the sinuous mode, suggesting that the sinuous mode is essentially a gyrotactic instability in the downflowing suspensions. This mechanism is seen to be strongly supplemented by the gradient in the cell-swimming vector field which yields the second largest contribution $\left(P_{e-\text { grad }}\right.$ in table 3). On the contrary, $P_{\text {inf }}$ and $P_{\text {diff }}$ are considerably smaller than $P_{\text {gyro }}$ and $P_{e-\text { grad }}$, indicating that the sinuous mode does not arise from the inflectional and the diffusionoriented mechanisms. It is also quite interesting to note that $P_{\text {grav }}$ is negative in this case. This implies that the gravitational mechanism plays a stabilizing role for the sinuous mode. In this respect, it is worth mentioning that the long wavelength sinuous mode in the falling line dense plume is stable at low Reynolds numbers (Lister 1987). Finally, this analysis gives an explanation why the sinuous mode is highly damped out for large flow rates. It is seen that, for $Q>0$, the damping given by $E_{n n}$ is much stronger than the production by $E_{n v}$. This suggests that the leading stabilisation mechanism of the sinuous mode is likely to be due to the enhanced damping of $L_{C}$ for $Q>0$. We further note that $E_{n v}$ remains almost the same for $Q>8$ (i.e. $E_{n v} \sim 1.5$ ) while $E_{n n}$ becomes more negative with an increase of $Q$. Since the largest contribution to $E_{n v}$ is made by $P_{\text {gyro }}$, which also does not increase very rapidly for $Q>8$ compared to that for $0<Q<8$, the less active nature of the gyrotactic instability mechanism for large $Q$ also plays a role in stabilisation of the sinuous mode (see also $\zeta_{1}$ and $\zeta_{2}$ at large $\Omega_{z 0}$ in figure $3 a$ ).

The contributions of each term to the varicose mode are given in table 4. Similarly to the sinuous mode, $E_{n v}+E_{n n}$ roughly gives the growth rate $\omega_{i}$ and $E_{o s}$ and $E_{v n}$ are balanced with each other. However, the contribution of each of the instability production terms for the varicose mode appears to be quite different from that of the sinuous mode. First, none of the production terms contribute to the instability at zero flow rate $(Q=0)$, explaining why it is independent of Ri. However, on increasing the flow rate, each term begins to play a role in generating the instability. In particular, the largest contribution 


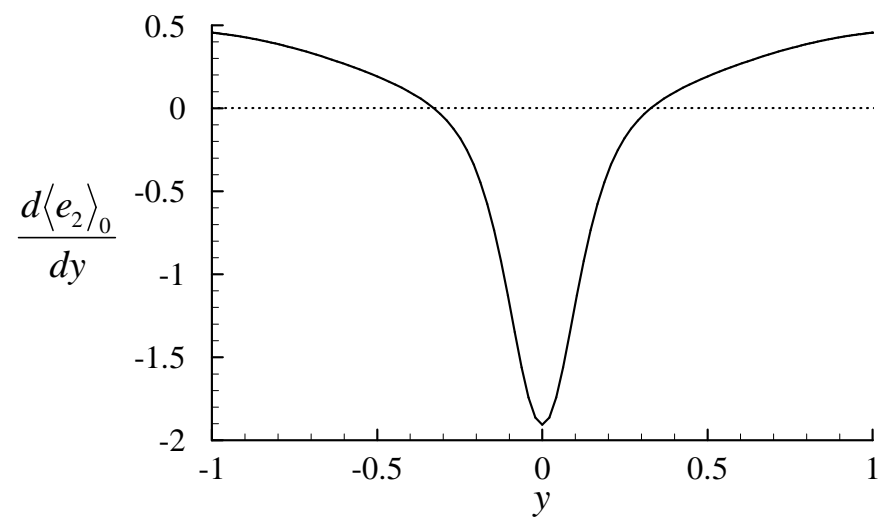

FiguRE 14. Profile of $d\left\langle e_{2}\right\rangle_{0} / d y$ along the horizontal direction for the reference parameters $(\mathrm{Ri}=90$ and $Q=4)$.

is made by $P_{e-g r a d}$, indicating that the varicose mode essentially originates from the gradient in the cell swimming vector field. None of the previous studies (e.g. Pedley \& Kessler 1992) have considered this mechanism, and it suggests that the varicose mode is a consequence of the following simplified dynamics:

$$
\frac{\partial n^{\prime}}{\partial t} \sim-\frac{d\left\langle e_{2}\right\rangle_{0}}{d y} n^{\prime}
$$

Plotting $d\left\langle e_{2}\right\rangle_{0} / d y$ as in figure 14 indeed confirms that this mechanism yields the unstable varicose instability (note that $d\left\langle e_{2}\right\rangle_{0} / d y<0$ along $y=0$ ). However, it should also be emphasised that the other mechanisms related to gyrotaxis and gravity also play some role in supporting the varicose instability. Table 4 shows that the gyrotactic mechanism also strongly cooperates in generating the varicose mode by making the second largest contribution. Also, the gravitational mechanism makes a small contribution to the varicose-mode generation, unlike the sinuous mode, consistent with Lister (1987) where the long wavelength varicose mode is found to be unstable in a range of low Reynolds numbers. Contributions of both the inflectional and the diffusion-oriented instability mechanisms to the varicose mode are found to be negligible as they are to the sinuous one. This suggests that the co-existence of the inflection point in the flow field and the blip instability (the varicose mode in the present study) reported by Kessler (1986) is likely to be just a coincidence as the inflectional instability mechanism would be suppressed by the strong fluid viscosity at low Reynolds numbers. Finally, the analysis also provides the stabilisation mechanism of the varicose mode at large flow rates. It is first interesting to note that $E_{n n}$ changes only a little as the flow rate is increased: i.e. $E_{n n} \simeq-0.43 \sim-0.48$ for $Q>4$. On the other hand, $E_{n v}$ slowly decreases as the flow rate is increased. Since the largest contribution to $E_{n v}$ is given by $P_{g y r o}$, this indicates that the gyrotactic instability mechanism diminishes on increasing $Q$. Furthermore, $P_{e-g r a d}$ is also seen to grow more slowly at large flow rate $(8<Q<16)$ than at small flow rate $(0<Q<8)$. These observations suggest that the stabilization of the varicose mode on increasing $Q$ is likely to be due to disruption of the gyrotactic nature of the cells caused by the increased local vorticity (or shear).

\subsection{Outlook}

While performing the present analysis, a few difficulties that we have had suggests that the model (2.1) still needs to be improved further in a number of respects. First, improving 
the simple expression (2.3) for the cell diffusivity tensor is very desirable as already mentioned. The generalised Taylor dispersion theory would be a good starting point in this regard as it has recently been shown to provide a reliable prediction for self-propelling particles with a constant rotational diffusivity (e.g. Bearon et al. 2011; Croze et al. 2013). However, it is not evident yet whether it would also provide a good description for the dispersion of 'real' cells. In this respect, experiments which examine the cell dispersion in the presence of flow would be necessary for making theoretical progress. Another important difficulty in handling the present model (2.1) is its dimension: (2.1) is composed of a set of six-dimensional equations owing to its dependence on $t, \mathbf{x}$ and $\mathbf{e}$. This feature would require extremely expensive computational cost, even with modern computing power, particularly if one is to solve (2.1) directly without any approximation. Therefore, a reliable approach or theory for approximating the statistical moments such as $\langle\mathbf{e}\rangle$ and $\langle$ ee $\rangle$ without directly tackling the Smoluchoski equation or the Fokker-Planck equation (2.2c) needs to be developed. Such an approximation was, in fact, made for a suspension of passive Brownian particles by e.g. Hinch \& Leal (1976). Finally, the present model is built upon the assumption that the suspension is dilute, thus it is desirable to develop a continuum model for dense suspensions. Such a model has recently been proposed by Ezhilan et al. (2013), in which the steric interaction in dense suspensions is modeled such that contacts between nearby particles cause them to align locally. Together with a set of experiments, developing and verifying such a mathematical model therefore also remains an important target of future work.

\section{Acknowledgements}

We are grateful to O. A. Croze for discussions on this work. Y.H. is supported by the European Commission through a Marie Curie fellowship.

\section{REFERENCES}

Bearon, R. N., Bees, M. A. \& Croze, O. A. 2011 The spatial distribution of gyrotactic swimming micro-organisms in laminar fow fields. J. Fluid Mech. 680, 602-635.

Bearon, R. N., Bees, M. A. \& Croze, O. A. 2012 Biased swimming cells do not disperse in pipes as tracers: a population model based on microscale behaviour. Phys. Fluids 24, 121902 .

Bees, M. A. \& Hill, N. A. 1997 Wavelengths of bioconvection patterns. J. Exp. Biol. 10, $1515-1526$

Bees, M. A. \& Hill, N. A. 1998 Linear bioconvection in a suspension of randomly swimming, gyrotactic micro-organisms. Phys. Fluid 10 (8), 1864-1881.

Bees, M. A. \& HiLl, N. A. 1999 Non-linear bioconvection in a deep suspension of gyrotactic swimming micro-organisms. J. Math. Biol. 38, 135-168.

Childress, S., Levandowsky, M. \& Spiegel, E. A. 1975 Pattern formation in a suspension of swimming micro-organisms. J. Fluid Mech. 69, 591-613.

Croze, O. A., Sardina, G., Ahmed, M., Bees, M. A. \& Brandt, L. 2013 Dispersion of swimming algae in laminar and turbulent channel flows: consequences for photobioreactors. J. Royal Soc. Interface 10, 20121041.

Denissenko, P. \& Lukaschuk, S. 2007 Velocity profiles and discontinuities propagation in a pipe flow of suspension of motile microorganisms. Phys. Lett. A 362, 298-304.

Drazin, P.G. \& Reid, W.H. 1981 Hydrodynamic Stability. Cambridge Univ. Press.

Ezhilan, B., Shelley, M. J. \& Saintillan, D. 2013 Instabilities and nonlinear dynamics of concentrated active suspensions. Phys. Fluids 25, 070697.

Ghorai, S. \& HiLL, N. A. 1999 Development and stability of gyrtatic plummes in bioconvection. J. Fluid Mech. 400, 1-31. 
Ghorai, S. \& Hill, N. A. 2000 Periodic arrays of gyrotactic plumes in bioconvection. Phys. Fluids 12, 5-22.

Hill, N. A. \& Bees, M. A. 2002 Taylor dispersion of gyrotactic swimming micro-organisms in a linear flow. Phys. Fluid 14, 2598-2605.

Hill, N. A. \& Pedley, T. J. 2005 Bioconvection. Fluid Dyn. Res. 37, 1-20.

Hill, N. A., Pedley, T. J. \& Kesssler, J. O. 1989 Growth of bioconvection patterns in a suspension of gyrotactic micro-organisms in a layer of finite depth. J. Fluid Mech. 208, 509-543.

Hinch, E. J. \& LeAL, L. G. 1976 Constitutive equations in suspension mechanics. part 2. approximate forms for a suspension of rigid particles affected by brownian rotations. $J$. Fluid Mech. 76, 187-208.

Hwang, Y. \& Pedley, T. J. 2014 Bioconvection under uniform shear: linear stability analysis. J. Fluid Mech. 738, 522-562.

KEsSLER, J. O. 1984 Gyrotactic buoyant convection and spontaneous pattern formation in algal cell cultures. In Non-Equilibrium Cooperative Phenomena in Physics and Related Fields (ed. M. G. Verlarde), pp. 241-248. Plenum.

Kessler, J. O. 1985a Co-operative and concentrative phenomena of swimming microorganisms. Contemp. Phys. 26, 147-166.

Kessler, J. O. $1985 b$ Hydrodynamics focusing of motile algal cells. Nature 315, 218-220.

Kessler, J. O. 1986 Individual and collective dynamics of swimming cells. J. Fluid Mech. 173, 191-205.

Lister, J. R. 1987 Long-wavelength instability of a line plume. J. Fluid Mech. 175, 413-428.

Malena, A. \& Frankel, I. 2003 Generalized Taylor dispersion in suspensions of gyrotactic swimming micro-organisms. J. Fluid Mech. 490, 99-127.

Pedley, T. J. 2010 a Collective behaviour of swimming micro-organisms. Exp. Mech. 50, 12931301.

Pedley, T. J. $2010 b$ Instability of uniform microorganism suspensions revisited. J. Fluid Mech. 647, 335-359.

Pedley, T. J., Hill, N. A. \& Kessler, J. O. 1988 The growth of bioconvection patterns in a uniform suspension of gyrotactic micro-organisms. J. Fluid Mech. 195, 223-237.

Pedley, T. J. \& Kessler, J. O. 1990 A new continuum model for suspensions of gyrotactic micro-organisms. J. Fluid Mech. 212, 155-182.

Pedley, T. J. \& Kessler, J. O. 1992 Hydrodynamic phenomena in suspensions of swimming micro-organisms. Annu. Rev. Fluid Mech. 24, 313-358.

Pope, S. B. 2000 Turbulent flows. Cambridge, UK: Cambridge Univ. Press.

Sмith, M. K. 1989 The axisymmetric long-wave instability of a concentric two-phase pipe flow. Phys. Fluids A 1, 494-506.

Vladimirov, V. A., Denissenko, P. V., Pedley, T. J., We, M. \& Zakhidova, I. S. 2004 Measurement of cell velocity distributions in populations of motile algae. J. Exp. Biol 207, 1203-1216.

Weideman, J. A. C. \& Reddy, S. C. 2000 A MATlab Differentiation Matrix Suite. ACM Trans. Math. Soft. 26, 465-519. 